\title{
El Sistema de Epiciclos y Deferentes de Ptolomeo:
} una reconstrucción*

\author{
The Ptolemy Epicycle and Deferent System: A Reconstruction \\ Christián C. Carman ${ }^{\dagger}$
}

\begin{abstract}
Resumen
En este trabajo se ofrece una primera versión de una reconstrucción estructuralista de la teoría de epiciclos y deferentes propuesta por Claudio Ptolomeo en el Almagesto para explicar el movimiento planetario, con especial interés en aquellos aspectos que permitan llegar al cálculo de la distancia de los planetas ofrecida el mismo Ptolomeo en Las hipótesis planetarias.
\end{abstract}

Palabras clave: epiciclo - deferente - ecuante - reconstrucción - metateoría estructuralista

\begin{abstract}
This paper offers a first version of a structuralist reconstruction of the theory of epicycles and deferents proposed by Claudius Ptolemy in the Almagest to explain planetary motion, with special interest in those aspects that allow us to calculate the distance of the planets offered by Ptolemy himself in Planetary Hypotheses.
\end{abstract}

Keywords: epicycles - deferents - equator - reconstruction - structuralism

${ }^{*}$ Recibido: 3 de febrero de 2013. Aceptado con revisiones: 19 de mayo de 2013.

† Universidad Nacional de Quilmes (UNQ)/Consejo Nacional de Investigaciones Científicas y Técnicas (CONICET), Argentina. Para contactar al autor, por favor, escribir a: ccarman@gmail.com.

Metatheoria 6(1)(2015): 47-72. ISSN 1853-2322. eISSN 1853-2330.

(C) Editorial de la Universidad Nacional de Tres de Febrero. Publicado en la República Argentina. 


\section{Introducción}

Es sin duda extraño que una de las teorías que ha estado entre las más citadas como ejemplo en toda la epistemología del siglo XX, como lo es la astronomía ptolemaica, no haya sido aun reconstruida por la concepción estructuralista. ${ }^{1}$ En este trabajo me propongo remediar parcialmente tal carencia ofreciendo una primera versión de una reconstrucción estructuralista si no de toda la astronomía ptolemaica, al menos de la teoría de epiciclos y deferentes propuesta por Ptolomeo para explicar el movimiento planetario, con especial interés en aquellos aspectos que permitan llegar al cálculo de la distancia de los planetas ofrecida por Ptolomeo en Las hipótesis planetarias. Supondremos a lo largo del escrito un conocimiento medianamente detallado de la teoría ${ }^{2}$ e iremos haciendo referencia, cuando lo creamos oportuno, a las obras de Ptolomeo. ${ }^{3}$ En la primera parte reconstruiremos el elemento teórico básico, primero los elementos del núcleo teórico -los modelos potenciales, modelos, modelos parciales, condiciones de ligadura y vínculos interteóricos- y luego las aplicaciones propuestas, para llegar a la aserción empírica de la teoría. En la segunda parte, ofreceremos algunas importantes especializaciones para los planetas interiores, exteriores y el Sol, para concluir con una representación de la red teórica. En los apéndices brindaremos algunas demostraciones y definiciones que, colocadas en el cuerpo principal, habrían entorpecido la lectura ya de por sí enmarañada. De más está decir que no pretende ser una reconstrucción completa ni perfecta, quedarán muchas partes importantes por reconstruir -por ejemplo, las leyes especiales de la Luna, la teoría de los eclipses, etc.- y muchas partes aquí reconstruidas probablemente luego serán perfeccionadas. ${ }^{4}$ Creemos que la reconstrucción ofrece un material sumamente fecundo desde el punto de vista metateórico, pero la reflexión epistemológica la dejaremos para trabajos posteriores.

\section{El elemento teórico básico del Sistema de Epiciclos y Deferentes de Ptolomeo}

Aquí se introducen los distintos componentes del núcleo teórico del Sistema de Epiciclos y Deferentes de Ptolomeo K(SED) -la clase de los modelos potenciales $\mathbf{M}_{\mathrm{p}}(\mathrm{SED})$, la clase de los modelos $\mathbf{M}(\mathrm{SED})$, la clase de los modelos parciales $\mathbf{M}_{\mathrm{pp}}(\mathrm{SED})$, algunas condiciones de ligadura $\mathbf{C}(\mathrm{SED})$ y vínculos interteóricos $\mathrm{L}(\mathrm{SED})$ - y se caracteriza su campo de aplicaciones propuestas I(SED) y su afirmación empírica.

\subsection{El núcleo teórico del Sistema de Epiciclos y Deferentes de Ptolomeo}

\subsubsection{Los modelos potenciales de SED}

Se comienza la reconstrucción a partir de la caracterización de los modelos potenciales, constituido por estructuras que satisfacen ciertas condiciones estructurales (los axiomas impropios) para ciertos

\footnotetext{
${ }^{1}$ El único trabajo que existe sobre el tema, dentro de esta tradición, es Heidelberg (1976), pero dista mucho de ser una reconstrucción.

${ }^{2}$ La teoría ptolemaica está detalladamente explicada en Pedersen (1974) y Neugebauer (1975); una introducción más sencilla a toda la astronomía antigua puede verse en Evans (1998). La mejor traducción al inglés del Almagesto es Toomer (1998). La edición griega clásica es Heiberg (1898-1903). Otra obra que trabajaremos será Las hipótesis planetarias de Ptolomeo. La edición griega, nuevamente, es de Heiberg (1907), aunque está incompleta; el texto faltante en su edición árabe y traducción al inglés está en Goldstein (1967) y una traducción completa al castellano puede encontrarse en Pérez Sedeño (1987).

3 En el caso del Almagesto simplemente indicaremos la cita de la obra de Heiberg, como es habitual.

${ }^{4}$ Agradezco muy particularmente a Pablo Lorenzano, sin su paciente ayuda hubiera sido imposible realizar este trabajo; también va mi agradecimiento a todos los participantes del V Encuentro Iberoamericano sobre Metateoría Estructuralista por sus comentarios, en particular a José Díez, y a los miembros del Seminario Semanal Permanente en Filosofía de la Ciencia (IEC/UNQ) en donde expuse una versión anterior de este trabajo.
} 
conceptos (los conceptos de la teoría), que expresan el marco conceptual de la teoría, y de las que tiene sentido preguntarse si son modelos actuales de la teoría.

\section{Definición 1:}

$\mathbf{M}_{\mathrm{p}}(\mathrm{SED}): x=\left\langle P, P l, T, \mathrm{O}, \mathbb{R}, \mathbb{N}\right.$, pun, co, $r, c, \omega, p m, \lambda, \beta, \sigma, b r{ }^{m}$, SOM, pm som $\rangle$ es un sistema de epiciclos y deferentes de Ptolomeo potencial $\left(x=\left\langle P, P l, T, O, \mathbb{R}, \mathbb{N}\right.\right.$, pun, co, $r, c, \omega, p m, \lambda, \beta, \sigma, b r,{ }^{m}$, $\left.\left.\mathrm{SOM}, p m_{\mathrm{som}}\right\rangle \in \mathbf{M}_{\mathrm{p}}(\mathrm{SED})\right)$ syss

(1) $P$ es un conjunto no-vacío

(2) $\mathrm{Pl}$ es un conjunto finito, no-vacío

(3) $T=\left\langle\mathbb{R}^{+},<\right\rangle$

(4) pun: $\mathrm{Pl} \rightarrow P$

(5) $\quad$ co: $P \times P \times P \times P \times P \times T \rightarrow \mathbb{R} \times \mathbb{R}_{(0.360]} \times \mathbb{R}_{(0.360]}$

(6) Def.: $O \subset P \& p_{1} \in O$ syss $\forall t \in T \& \forall g \in \mathbb{R}_{(0-360)}, \exists p_{2}, p_{3}, p_{4}, p_{5} \in P \& \exists h \in \mathbb{R}_{(0-360]} \& \exists s \in \mathbb{R}^{+}$: $c o\left(p_{1}, p_{2}, p_{3}, p_{4}, p_{5}, t\right)=(s, g, h)$

(7) Def.: $r_{(0)}=_{\text {def }} s$ syss $s \in \mathbb{R}^{+} \& o_{1} \in O \& \forall t \in T \& \forall p_{1} \in o_{1} \& \forall g \in \mathbb{R}_{(0.360)}, \exists p_{2}, p_{3}, p_{4}, p_{5} \in P \&$ $\exists h \in \mathbb{R}_{(0.360):} \operatorname{co}\left(p_{1}, p_{2}, p_{3}, p_{4}, p_{5}, t\right)=(s, g, h)$

(8) Def.: $c_{(0, t)}{ }_{\text {def }} z$ syss $z \in P \& o_{1} \in O \& \forall t \in T, p_{1} \in o_{1}, g \in \mathbb{R}_{(0-360)}, \exists p_{3}, p_{4}, p_{5} \in P \& \exists h \in \mathbb{R}_{(0-360)}$ $\& \exists x \in \mathbb{R}^{+}: c_{0}\left(p_{1}, z, p_{3}, p_{4}, p_{5}, t_{1}\right)=(x, g, h)$

(9) Def.: $\omega: O \times P \times T \rightarrow \mathbb{R}_{(0-360]} \& \omega_{(01, \mathrm{x}, \mathrm{t})}=w$ syss $o_{1} \in O \& x \in P \& t \in T \& \exists o_{y} \in O \& c_{(o y, t)}=$ $c_{(o 1, t)} \& r_{(o y)}<r_{(o 1)}: x \in o_{y} \cup c_{(01, t)}$

(10) Def.: pm(o,t) ${ }_{\text {def }} m$ syss $o_{1} \in O \& t \in T \& m \in o_{1} \& \exists x \in \mathbb{R}_{(0.360]} \& p_{3}, p_{4}, p_{5}, p_{6} \in P, \exists h \in \mathbb{R}_{(0.360]}$ : $c_{0}\left(m, c_{(0, t 0)}, p_{3}, p_{4}, p_{5}, t_{0}\right)=\left(r_{(0)}, x, h\right) \& c o\left(m, c_{(0, t)}, p_{3}, p_{4}, p_{5}, t\right)=\left(r_{(0)}, \omega\left(o, p_{6}, t\right) \cdot t+x, h\right)$

(11) $\lambda: P \times T \rightarrow \mathbb{R}_{(0-360]}, \lambda$ es continua entre [0-360]

(12) $\beta: P \times T \rightarrow \mathbb{R}_{(-90-+90)}$

(13) $\sigma: P \times T \times T \rightarrow\{-1,0,1\}$

(14) $b r: P l \times T \times T \rightarrow\{-1,0,1\}$

(15) ${ }^{m} \subseteq \mathrm{O} \times \mathrm{O} \& \forall t \in T: c_{(01, t)}=p m_{(02, t)}$

(16) $\operatorname{SOM}_{p l} \subseteq O \times O: o \in \operatorname{def}_{\operatorname{def}} \mathrm{SOM}_{p l}$ syss $o \in O \& p l \in P \& n \in \mathbb{N} \& o_{1}, o_{2}, \ldots, o_{n} \in O \& o_{1}{ }^{m} o_{2} \& o_{2}{ }^{m}$ $o_{3} \& 0_{3}{ }^{m} 0_{4} \& \ldots \& o_{n-1}{ }^{m} o_{n} \&\left(o=o_{1} \vee 0=o_{2} \vee 0=o_{3} \vee \ldots \vee 0=o_{n-1} \vee 0=o_{n}\right)$

(17) Def.: $p m_{(\mathrm{som}, \mathrm{t})}={ }_{\text {def }} k$ syss $o_{x} \in \mathrm{SOM}, \nexists o_{n} \in \mathrm{SOM}: o_{n}{ }^{m} o_{x} \& \forall t \in T, k=p m_{(\mathrm{ox}, \mathrm{t})}$.

\section{Axiomas de interpretación:}

\section{(1), (2) y (3): Puntos, planetas y tiempo}

Los conjuntos base serán $P, P l, T$. $P$ es el conjunto de puntos. $P l$ es el conjunto de los planetas, es un conjunto no vacío y con, al menos, siete elementos (en el que no se incluye, evidentemente, a la Tierra): Luna, Mercurio, Venus, Sol, Marte, Júpiter y Saturno. T es el conjunto de los instantes de tiempo. Diremos que es está compuesto por un conjunto de instantes representados por los números reales positivos a los que se le aplica una relación de precedencia que los ordena, tal cual está presentado en Balzer (1997, 155-159). Para simplificar, los subíndices presuponen ya el orden, así, por ejemplo, $t_{1}$ es anterior a $t_{2}$. Está representado por los reales positivos porque en el sistema de Ptolomeo existe un $t=0$ que corresponde al primer día del mes de Toth del primer año de la era de Nabonassar, al mediodía de Alejandría (26 de febrero de -746) [H256], expresado en días julianos: 1.448.638.

\section{(4): Puntación}

Como muchas funciones tendrán en su dominio puntos, necesitamos poder tratar a los planetas como puntos. La función pun (puntación) atribuye a cada planeta un punto determinado, a saber, el centro de su esfera.

\section{(5): Coordenadas polares}

Las coordenadas polares tienen en el dominio cinco puntos y el tiempo. El primero de estos puntos 
$\left(p_{1}\right)$ es el punto del cual se predicará la coordenada (el que será ubicado), el segundo ( $\left.p_{2}\right)$ será el polo (el eje de las coordenadas) y el segundo con el tercero $\left(p_{3}\right)$ formarán una recta que será aquella desde la cual se midan los ángulos. Es decir, si el primer punto (el que queremos ubicar) se encuentra alineado con los otros dos, su ángulo será o bien 0 si el polo no se encuentra entre los otros dos puntos, o bien 180 si sí lo está. Los tres puntos formarán un plano, que será el plano sobre el que esté apoyada la órbita. ${ }^{5}$ Los otros dos puntos ( $p_{4}$ y $p_{5}$ ) junto con el polo $\left(p_{2}\right)$ formarán otro plano, de referencia. La función co da como resultado tres números reales: el primero es el radio $s$ (la distancia entre el punto que hace de polo y el punto que queremos ubicar), el segundo da el ángulo $g$ que ha barrido ese punto sobre el plano de la órbita y el tercero $h$ es también un ángulo (que será constante) que indica la inclinación del plano formado por los tres puntos del dominio respecto del plano de referencia, formado por los otros tres puntos. Si el radio permanece constante, la órbita descrita será circular (ver figura 1).

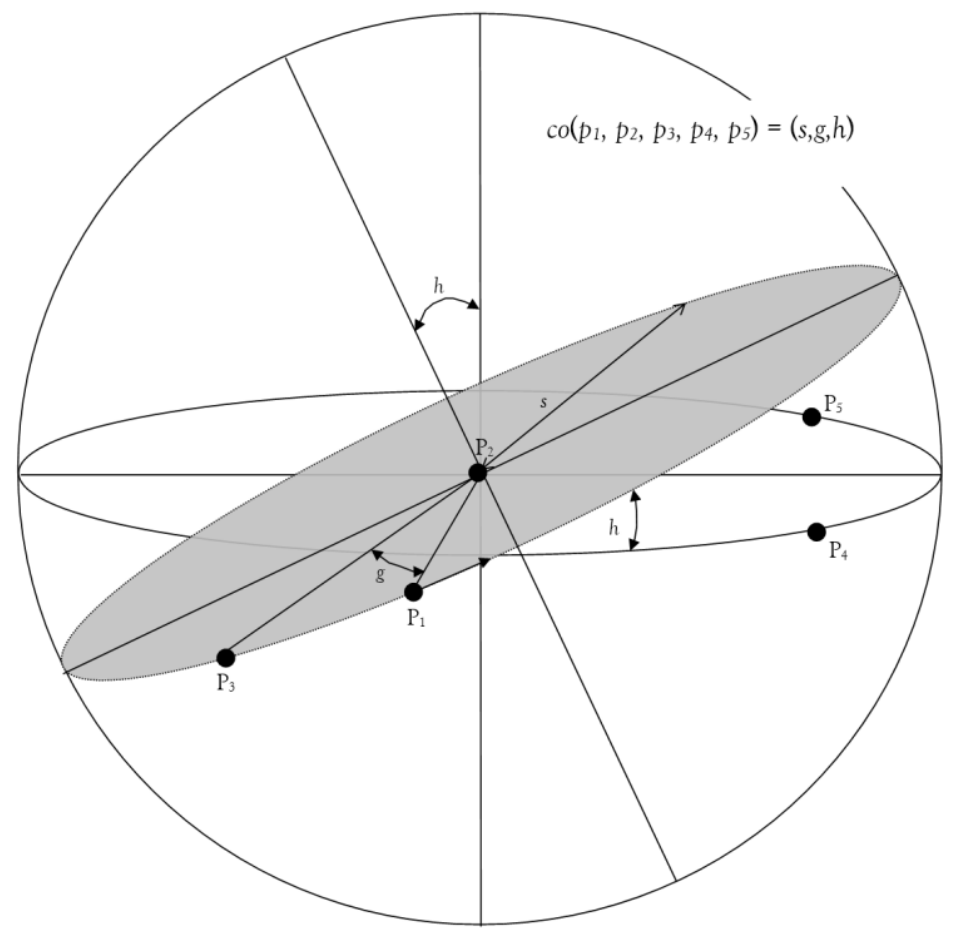

Figura 1.

\section{(6): Órbitas}

$\mathrm{O}$, el conjunto de las órbitas, es un subconjunto de los puntos. Se trata de todos los puntos que forman la circunferencia. La hemos definido mediante las coordenadas polares. El axioma 6 afirma que $\mathrm{O}$ es un subconjunto de los puntos y un determinado punto $\left(p_{1}\right)$ pertenece a la órbita si en todo tiempo $(t)$ y para todo ángulo $(g)$, las coordenadas polares de ese punto $\left(p_{1}\right)$ están determinadas por un radio determinado (s) que no varía -y eso garantiza la circularidad de la órbita-, cualquier ángulo $g-y$ eso garantiza la completitud de la órbita- y un tercer ángulo constante $(h)$ que garantiza que la órbita se mantiene sobre un mismo plano. ${ }^{6}$ A las órbitas se le atribuyen ciertas funciones y tienen ciertos términos definidos.

\footnotetext{
${ }^{5} \mathrm{Si}$ los tres puntos estuvieran alineados no formarían un plano, pero como el punto que queremos ubicar es un punto que se desplaza, necesariamente en algún momento no estarán alineados.

${ }^{6}$ No debe confundirse la órbita de un planeta con su trayectoria. La trayectoria es el trazo formado por el planeta al desplazarse que no tiene por qué ser circular, mientras que la órbita es una construcción teórica con movimiento necesariamente circular. Puede observarse una trayectoria, no una órbita.
} 


\section{(7): El radio de la órbita}

Cada órbita tiene, en primer lugar, un radio $(r)$, expresado por un número real positivo. El axioma 7 dice, simplemente, que el radio es el primer componente de la imagen de las coordenadas polares de cualquier punto de esa órbita.

\section{(8): Centro de la órbita}

Cada órbita tiene, en segundo lugar, un centro (c), alrededor del cual se trazará la circunferencia. Lo que dice el axioma 8 es que ese punto está definido como el segundo componente del dominio de las coordenadas polares de cualquier punto de la órbita. Como el centro puede ir variando (si, por ejemplo, es el centro de un epiciclo), está en función del tiempo.

\section{(9): Velocidad angular}

Cada órbita tiene una velocidad angular $(\omega)$, expresada en grados, que se mide tomando algún punto interior a la órbita como eje. Como veremos luego, la identificación o no de este punto con el centro de la órbita servirá para discriminar si se ha utilizado o no el ecuante. La velocidad angular está en función del tiempo pues, si bien es sabido que Ptolomeo y, en general, la astronomía antigua buscaba velocidades constantes, el hecho de que las buscaran implica que existía la posibilidad de que no las hubiera. De hecho, el ingenioso dispositivo del punto ecuante, como veremos luego, surgió para poder hacer constante una velocidad que no lo era. Ahora bien, el punto desde el cual se mide la velocidad angular tiene que cumplir con el requisito de ser interior a la órbita. Este criterio puede expresarse diciendo que ese punto es un elemento de la unión del punto que forma el centro de la órbita y el conjunto formado por todos los puntos de las órbitas que tienen el mismo centro y un radio menor que la órbita de la que se medirá la velocidad angular. ${ }^{7}$

\section{(10): Punto móvil de una órbita}

pm es, intuitivamente, un punto móvil. En realidad no es un punto que se va moviendo, sino que el punto móvil va siendo puntos distintos. Este punto será aquél en el que se ubique o bien el planeta (si es la última órbita) o bien el centro de alguna otra órbita y se desplaza por la órbita con la velocidad angular $\omega$. El axioma 10 sostiene que el punto móvil pertenece al subconjunto de los puntos que forman una determinada órbita y las coordenadas polares de ese punto, tomando como polo al centro de la órbita en un determinado instante de tiempo, será igual al radio de la órbita como primer término y al ángulo que surja del producto de la velocidad angular (medida desde un determinado punto p6) por el tiempo más el ángulo del tiempo inicial -que ha sido definido $\operatorname{como} x$, siendo $x$ el segundo componente de la imagen de la co de $m$ cuando el tiempo es $t_{0}$. Nuevamente, hay un punto (p3) que es el tercer punto de la coordenada polar que permite determinar -junto con el centro de la órbita el ángulo $0 \mathrm{y}$, por supuesto, otros dos puntos ( $p_{4}$ y $p_{5}$ ) que junto con el polo determinarán el plano sobre el que gira la órbita $(h)$.

\section{(11), (12) y (13): Longitud y latitud celeste, distancia Tierra-Planeta}

Cualquier elemento del conjunto $P$ (cualquier punto) puede ser ubicado en el cielo a través de dos coordenadas. Para la ubicación de los planetas, Ptolomeo utilizó la Longitud Celeste $(\lambda)$ y la Latitud Celeste $(\beta)$. La primera $(\lambda)$ mide el ángulo que hay entre el punto en el que la eclíptica se cruza con el ecuador celeste (el equinoccio de primavera), el centro de la Tierra y el punto de la eclíptica más cercano al punto que queremos determinar (ángulo TOB en la figura 2). El ángulo se encuentra en el plano de la eclíptica. $\beta$, en cambio, mide el ángulo entre el punto que queremos ubicar, el centro de la Tierra y el punto de la eclíptica más cercano a aquel punto (SOB). El ángulo es perpendicular al plano de la eclíptica. Estas coordenadas, como miden ángulos, son expresadas en grados. Los axiomas 11 y 12 afirman, entonces, que tanto $\lambda$ como $\beta$ son funciones que tienen en su dominio a un punto y a un instante de tiempo y dan, como resultado, en el caso de la longitud un número real entre 0 y 360 (este último excluido porque en él vuelve a 0), y entre -90 y 90 en el caso de la latitud, que deben ser

\footnotetext{
${ }^{7}$ Agradezco a José Díez la sugerencia de expresar el interior de una órbita de esta manera tan elegante.
} 
interpretados como ángulos. La función $\lambda$ es continua entre [0 y 360], es decir, en todos los valores menos en el 0 porque en él la función pega un salto. Afirmarlo es necesario porque luego definiremos la retrogradación de los planetas en función de la derivada de la longitud.

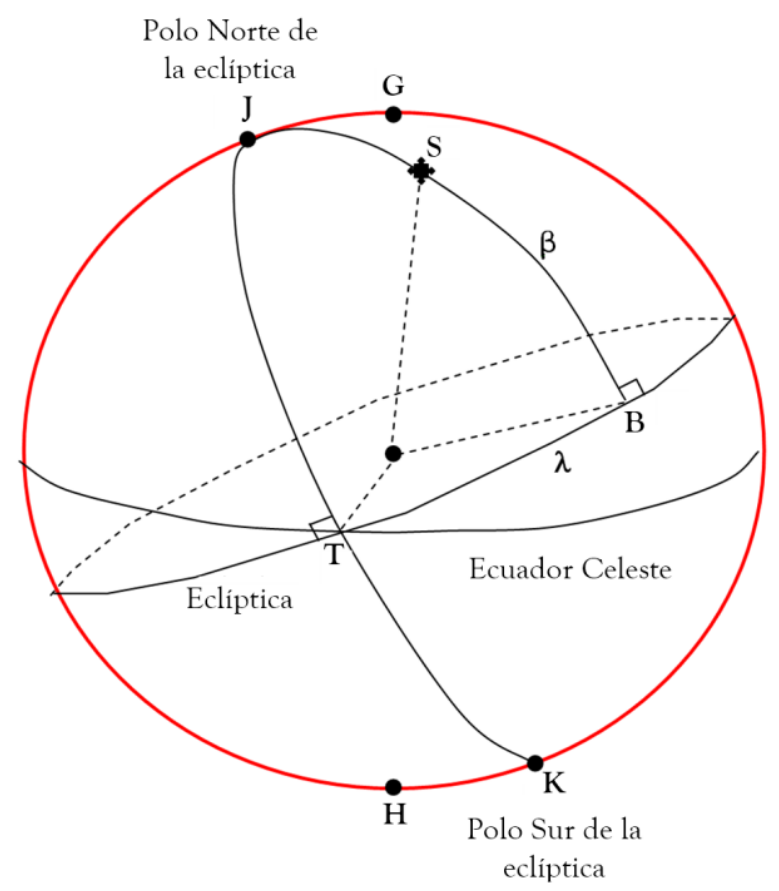

Figura 2.

Estas dos coordenadas son suficientes para determinar la posición de cualquier punto en el mapa celeste, pero no nos dan la profundidad, es decir, la distancia entre ese punto y el centro de la Tierra. Se podría considerar que no es necesario incluir la profundidad en una reconstrucción de SED, después de todo, el sistema de epiciclos y deferentes fue introducido para poder ubicar a los planetas sobre el mapa celeste y, para ello, son suficientes las dos primeras coordenadas introducidas. Pero también es cierto que el cambio en el brillo de los planetas (interpretado como cambio de distancia entre la Tierra y el planeta) fue una especie de experimento crucial en contra del sistema de Eudoxo y a favor del de epiciclos y deferentes. Por lo tanto, el dar cuenta del cambio de brillo en un planeta es relevante en el sistema y es conveniente que sea rescatado en la reconstrucción. Pero téngase presente que esta profundidad (distancia Tierra-Planeta) aquí es considerada exclusivamente como un concepto comparativo que servirá para dar cuenta de los cambios de brillo. Si hay más brillo, estará más cerca, si hay menos, estará más lejos, pero el sistema no atribuye números con significado físico a esas distancias. Aquí, simplemente, el 1 significa que hubo un incremento en su distancia, el 0 que ha permanecido igual y el -1 que se ha acercado, no importa la cantidad, respecto del instante anterior considerado.

$\mathrm{Ni}$ siquiera se trata de un término comparativo como puede serlo, por ejemplo, la dureza, que permite comparar la dureza de dos materiales distintos. Aquí sólo tendrá sentido decir que un mismo planeta en un tiempo posterior respecto de otro, está más, menos o igual de lejos, pero no es posible hacer comparaciones entre planetas. Por eso, $(\sigma)$ será expresada como una función entre un punto y dos instantes de tiempo. Ello dice el axioma 13.

\section{(14): Brillo}

El brillo tiene la misma naturaleza lógica que $\sigma$. Pero en este caso una relación entre un planeta y dos instantes de tiempo y permite comparar el brillo de un mismo planeta en dos instantes de tiempo. Evidentemente, el 1 indicará un aumento de brillo, el 0 que no ha cambiado y el -1 que ha 
disminuido.

\section{(15): La relación de montaje}

Ahora contamos con todos los elementos para poder describir una órbita. Pero el SED es, justamente, un sistema que encastra de una manera particular varias órbitas. Debemos, por lo tanto, introducir una relación que vincule órbitas entre sí que llamaremos función de montaje $\left({ }^{m}\right)$. La forma de montar las órbitas es muy precisa: una órbita $b$ está montada sobre otra órbita $a\left(a^{m} \mathrm{~b}\right)$ si y sólo si la posición del centro de la órbita $b$ es igual a la posición del punto móvil de la órbita $a$. Ello es lo que establece el axioma. En él se dice que una órbita $\left(\mathrm{O}_{2}\right)$ está montada sobre otra órbita $\left(\mathrm{O}_{1}\right) \mathrm{si}$, dado cualquier instante de tiempo, el centro de $\mathrm{O}_{2}$ coincide con el punto móvil de o1.

\section{(16): El Sistema de Órbitas Montadas}

Podemos ahora definir el subconjunto de órbitas que están en una cadena de montaje como $\mathbf{S O M}_{\mathrm{pl}}$ (sistema de órbitas montadas para el planeta $p l$ ). Este subconjunto será un tuplo ordenado de órbitas montadas entre sí y una órbita $\left(o_{x}\right)$ pertenecerá a ese subconjunto si pertenece al conjunto de las órbitas que forman una cadena de montaje. ${ }^{8}$

\section{(17): El punto móvil de SED}

Finalmente debemos introducir el punto móvil del final del sistema de órbitas montadas que será el punto móvil de la última órbita que aparece en el SOM. Para definirlo, simplemente decimos que el punto móvil del SOM es igual, para cualquier instante, al punto móvil de aquella órbita que, perteneciendo al SOM, no es montada por ninguna otra, es decir, de la última.

\subsubsection{La clase de los modelos}

El conjunto de modelos de SED es un subconjunto de los modelos potenciales, cuyas estructuras satisfacen, además de los axiomas impropios, la ley fundamental del sistema de epiciclos y deferentes de Ptolomeo.

La ley fundamental de SED simplemente establece que para cada planeta existe un sistema de deferente y epiciclos tal que la longitud y latitud celeste de su punto móvil coincida con la longitud y latitud celeste observada del planeta y, además, que el brillo del planeta observado sea inversamente proporcional a la distancia Tierra-punto móvil del SOM $(\sigma)$ en cualquier instante de tiempo (como hay sólo tres valores, diremos que el brillo es igual a la distancia Tierra-punto móvil del SOM multiplicado por menos uno). Podría, por lo tanto, ser expresado de la siguiente manera:

\section{Definición 2:}

$\mathrm{M}(\mathrm{SED}): \operatorname{Si} x=\left\langle P, P l, T, \mathrm{O}, \mathbb{R}, \mathbb{N}\right.$, pun, co, $\left.r, c, \omega, p m, \lambda, \beta, \sigma, b r,{ }^{m}, \mathrm{SOM}, p m_{\text {som }}\right\rangle$ es un $\mathbf{M}_{\mathrm{p}}(\mathrm{SED})$, entonces $x$ es un sistema de epiciclos y deferente de Ptolomeo $(x \in \mathrm{M}(\mathrm{SED}))$ syss:

(1) para todo $y \in P l \&$ para $t \in T$, si pun $(y)=z \& \exists \operatorname{SOM}_{x} \& t_{1}, t_{2} \in T$, entonces

(a) $\lambda\left(p m_{\text {som }(y), t)}=\lambda(z, t)\right.$

(b) $\beta\left(p m_{\text {som(y) }}, t\right)=\beta(z, t)$

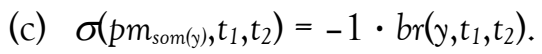

\subsubsection{La clase de los modelos parciales}

Para distinguir a los modelos potenciales de los parciales, tenemos que poder discriminar los conceptos SED-teóricos de los que no lo son. Recordemos que, intuitivamente un determinado concepto es teórico para una teoría si sólo puede ser determinado presuponiendo las leyes fundamentales de la teoría. Hagamos un breve análisis de los conceptos implicados en la teoría.

Está claro que el conjunto de puntos $(P)$ y de instantes de tiempo $(T)$ no forman parte de la teoría, ya que es evidente que no es necesario suponer el sistema de epiciclos y deferentes para determinar si

\footnotetext{
${ }^{8}$ Agradezco especialmente a Oscar Esquisabel su ayuda en la formalización de este axioma.
} 
algo es un punto o un instante de tiempo. La situación es un poco menos evidente con los conjuntos de planetas $(P l)$ y órbitas $(\mathrm{O})$. No cabe duda de que los planetas son esenciales a la teoría, pero forman parte de aquello que se quiere explicar, no aquello con lo que se explica y, para saber si algo es un planeta o no, no tengo que construir epiciclos y deferentes, por lo que tampoco se trata de un término SED-teórico. La situación es distinta en el caso de las órbitas, ya que sí se trata de algo propuesto para explicar y no de algo que se quiere explicar (recuérdese la distinción que hemos hecho entre órbita y trayectoria). Sin embargo, es claro que si bien es un término teórico, no lo es de ésta teoría en particular ya que para determinar si algo es o no una órbita no es necesario aplicar la teoría. Además, ha habido otras teorías que también utilizaron órbitas, como la propuesta de Eudoxo. Entonces, tampoco las $\mathrm{O}$ son conceptos SED-teóricos. Claramente tampoco lo son, por la misma razón, las funciones y términos definidos de las órbitas, su radio $(r)$, su centro $(c)$, su punto móvil ( $p m)$ y su velocidad angular $(\omega)$. La función puntación (pun) que atribuye a cada planeta un punto, tampoco supone la aplicación de la ley y lo mismo puede decirse de las coordenadas polares (co). Por otro lado, la longitud celeste $(\lambda)$ y la latitud celeste $(\beta)$ y los cambios de brillo $(b r)$ no presuponen la teoría para ser aplicadas, de hecho, es justamente lo que se quiere explicar con ella. Los cambios de distancia respecto de la Tierra $(\sigma)$ es propuesto para explicar los cambios de brillo, pero no es propio de la teoría, de hecho el que los cambios de brillo no correspondieran a los cambios de distancia fue la refutación de la propuesta de Eudoxo.

Los firmes candidatos para ser conceptos SED-teóricos son, entonces, la función montaje $\left(^{m}\right)$, el sistema de órbitas montadas ( $\mathrm{SOM}$ ) y el punto móvil de todo el sistema $\left(\mathrm{pm}_{\mathrm{som}}\right)$, pues lo propio de un sistema de epiciclos y deferentes es, justamente, la manera en que son montadas las órbitas y, en consecuencia, el sistema que varias de ellas forman. No tiene sentido hablar de ellos fuera de esta teoría, por lo tanto son los tres conceptos SED-teóricos.

\section{Definición 3:}

$\mathbf{M}_{\mathrm{pp}}(\mathrm{SED}): \quad y=\langle P, P l, T, \mathrm{O}, \mathbb{R}, \mathbb{N}, p u n, c o, r, c, \omega, p m, \lambda, \beta, \sigma, b r\rangle$ es un sistema de epiciclos y deferente de Ptolomeo parcial ( $y=\langle P, P l, T, O, \mathbb{R}, \mathbb{N}$, pun, co, r, c, $\left.\omega, p m, \lambda, \beta, \sigma, b r\rangle \in \mathbf{M}_{\mathrm{pp}}(\mathrm{SED})\right)$ syss existe un $x$ tal que:

(1) $x=\left\langle P, P l, T, O, \mathbb{R}, \mathbb{N}\right.$, pun $\left., c o, r, c, \omega, p m, \lambda, \beta, \sigma, b r,{ }^{m}, \operatorname{SOM}, p m_{\text {som }}\right\rangle \in \mathbf{M}_{\mathrm{p}}(\mathrm{SED})$

(2) $y=\langle P, P l, T, O, \mathbb{R}, \mathbb{N}$, pun, co, r, c, $\omega, p m, \lambda, \beta, \sigma, b r\rangle$.

\subsubsection{Condiciones de ligadura}

Si se asume una interpretación realista -como aparece sin duda en Las hipótesis planetarias- habría una relación entre los distintos SOM que los restringiría a través de una condición de ligadura. La idea intuitiva es que, dado un orden de planetas, como no existe el vacío, la distancia máxima alcanzada por el punto móvil de un determinado SOM tiene que coincidir exactamente con la distancia mínima alcanzada por el punto móvil del SOM inmediato posterior.

Lo primero que haremos, para tener ya a mano conceptos que iremos utilizando, es hacer una partición en el conjunto de órbitas, distinguiendo tres subconjuntos, el de deferentes (infra def. aux. 3), el de órbitas excéntricas (infra def. aux. 4) y el de epiciclos (infra def. aux. 5). Ya que el deferente será definido como la órbita cuya cantidad de revoluciones coincida, a la larga, con las del planeta, deberemos definir cantidad de revoluciones (infra def. aux. 2) y para hacerlo, primero debemos poder distinguir el subconjunto de instantes en los que la longitud es 0 (infra def. aux. 1). Luego definiremos el punto excéntrico (infra def. aux.7), es decir el centro inmóvil de una órbita cuando no coincide con el centro de la Tierra, para lo cual necesitamos destacar el centro de la Tierra (infra def. aux. 6) y, finalmente, el ecuante (infra def. aux. 8), es decir el centro desde el que se medirá la velocidad angular de una órbita cuando éste no sea el centro de la Tierra.

Con estos conceptos preliminares ya definidos, procederemos, en primer lugar, a dar el primer paso en la metrización de $\sigma$ (infra def. aux. 9) que nos permitirá considerar a la distancia desde la Tierra ya no 
sólo como un término comparativo entre dos instantes de tiempo sino como un término que, aunque relativo a cada SOM, nos permita hablar de distancias en sentido estricto. Como la distancia máxima y mínima es relativa a cada SOM, no necesitamos más. Con ese concepto ya podemos definir el momento del apogeo (infra def. aux. 10) y del perigeo (infra def. aux. 11), es decir, los instantes en los que un determinado SOM alcanza su máxima y mínima distancia respectivamente. Con los tiempos, ya podemos conocer las distancias relativas máximas (infra def. aux. 12) y mínimas (infra def. aux.13) y establecer, además, un método de determinación de la distancia relativa máxima (infra def. aux. 14) y mínima (infra def. aux. 15) y afirmaremos, además, que en cada SOM el radio del deferente es mayor a la sumatoria de todos los otros (infra def. aux. 16) y, luego estableceremos el grosor relativo de un SOM (infra def. aux. 17).

Pero la condición de ligadura establece relaciones entre distintos SOM, por lo que no habla de distancias relativas sino absolutas. Por ello, deberemos dar un segundo paso en la metrización de $\sigma$ (infra def. aux. 18) y definiremos las distancias absolutas máximas (infra def. aux. 19) y mínimas (infra def. aux.20) y el grosor absoluto de un SOM (infra def. aux. 21). Con todo ello más una función ordenadora de los planetas (infra def. aux. 22), regida por el criterio de determinación de la función ordenadora de los planetas (infra def. aux. 23), es posible definir la condición de ligadura.

\subsubsection{Definiciones auxiliares}

El conjunto de las órbitas, entonces, está compuesto por tres subconjuntos excluyentes y exclusivos. El conjunto de los deferentes (Def), el de los epiciclos (Epi) y el de las órbitas de los puntos excéntricos (Exc). En efecto, en el sistema propuesto por Ptolomeo, en el caso de Mercurio y la Luna, el centro del deferente, que es un punto excéntrico, gira sobre una pequeña órbita. La unión de Def, Epi, Exc es el conjunto de las órbitas. Estos tres subconjuntos son, de alguna manera, subconjuntos destacados. Ahora bien, estos subconjuntos destacados sí son SED-teóricos, aun cuando lo sean de un conjunto que no lo es, porque para identificarlos hace falta presuponer la teoría ya que su identidad dependerá del lugar que ocupen en la cadena de montaje que, como hemos visto, es un concepto SED-teórico. En efecto, como veremos en seguida, en la definición de cada uno de ellos aparece la función montaje.

Comenzaremos con la definición de deferente y, en función de él, definiremos a los restantes. Lo propio de un deferente, en el sistema de Ptolomeo, no es que esté centrado en la Tierra (ya que todos son excéntricos), tampoco que su centro sea fijo ni que sea la primera de las órbitas (ya que en el caso de Mercurio y la Luna, ellos giran sobre una pequeña órbita excéntrica). Hay, sin embargo, dos características que los distinguen, una más esencial que la otra. La primera es que siempre se trata de la órbita más grande. Así, podríamos definirla en función de su radio. Pero la característica más esencial es que su período coincide con el período medio del planeta. En largos períodos de tiempo, el planeta da la misma cantidad de vueltas sobre la eclíptica que la que da el punto móvil del deferente y esto porque, en el fondo, el deferente da cuenta de la velocidad media del planeta. Luego los epiciclos explicarán las desviaciones sobre esa velocidad.

Para ello primero definiremos una revolución, es decir, una vuelta de un punto móvil sobre su órbita. Intuitivamente, una vuelta se produce cuando el punto móvil de una determinada órbita vuelve a pasar por el mismo lugar.

Elijamos arbitrariamente como "lugar" desde el que se contarán las vueltas aquél en el que el ángulo que varía en las coordenadas polares de la órbita es 0 . Diremos, entonces, que la cantidad de vueltas es igual a la cantidad de elementos que tiene el conjunto de los tiempos en los que ese ángulo fue 0 , en un determinado período de tiempo. Afirmaremos, entonces, que la cantidad de vueltas es igual al cardinal (\#) del conjunto de los tiempos en los que ese ángulo fue 0 . En la definición de los instantes en los que la "longitud" es 0 , diremos que $t_{x}$ tiene que ser mayor que $t_{1}$ pero menor o igual que $t_{2}$ para que, si sucediera que justo en $t_{2}$ la longitud fuera 0 , entonces contaría como una vuelta

\footnotetext{
9 Colocamos "longitud" entre comillas porque no se trata, en sentido estricto, de la función longitud celeste $(\lambda)$ ya que ésta se mide desde el centro de la Tierra y la que estamos tratando aquí se mide desde el centro de la órbita. Por eso en la definición de estos instantes no aparece $\lambda$.
} 
más. Pero no contaremos como una vuelta que $t_{1}$ tenga longitud 0 para evitar que, si los dos la tienen, cuenten como dos vueltas cuando en realidad ha dado sólo una.

\section{Definición auxiliar 1 (subconjunto de instantes en los que la "longitud" es 0 ):}

(1) $\operatorname{Tr}_{(\mathrm{t} 1, \mathrm{t} 2, \mathrm{pm}(0))}=\left\{t_{r} / t_{r} \in T \& t_{1}, t_{2} \in T \& o \in O \& p_{3}, p_{4}, p_{5} \in P \& h \in \mathbb{R}_{(0-360)} \& s \in \mathbb{R}^{+} \& t_{1}<t_{r} \leq t_{2}\right.$ tal que co $\left.\left(p m_{(0, \mathrm{t})}, c_{(0, \mathrm{tx})}, p_{3}, p_{4}, p_{5}, t_{r}\right)=(s, 0, h)\right\}$

Luego definimos la función cantidad de revoluciones que será una función que devuelva la cantidad de instantes en los que el ángulo que varía fue 0 entre dos instantes determinados.

\section{Definición auxiliar 2 (cantidad de revoluciones):}

(1) $\operatorname{rev:} T \times T \times P \rightarrow \mathbb{N} / \mathrm{si} t_{1}, \mathrm{t}_{2} \in T \& o \in \mathrm{O}$, entonces $\operatorname{rev}_{(\mathrm{t} 1, \mathrm{t} 2, \mathrm{pm}(\mathrm{o}))}=\# \operatorname{Tr}_{(\mathrm{t} 1, \mathrm{t} 2, \mathrm{pm}(\mathrm{o}, \mathrm{t}))}$

Ahora simplemente decimos que deferente es aquella órbita que forma parte del SOM de un determinado planeta siempre que las revoluciones de su punto móvil coincidan a la larga (cuando el tiempo tiende a infinito) con las revoluciones del punto móvil del SOM, ya que éste coincide con el del planeta (si suponemos la ley fundamental). La última condición exige que el numeral del deferente, para todo planeta, sea uno, es decir que en cada SOM exista un y sólo un deferente:

\section{Definición auxiliar 3 (deferente):}

(1) $o_{1} \in \operatorname{Def}_{\mathrm{x}}$ syss $\exists x \in P l \& t_{1}, t_{2} \in T \& o_{1} \in \mathrm{SOM}_{\mathrm{x}}: \operatorname{rev}_{(\mathrm{t} 1, \mathrm{t} 2, \mathrm{pm}(\mathrm{SOMx}))} \rightarrow \operatorname{rev}(\mathrm{t} 1, \mathrm{t} 2, \mathrm{pm}(\mathrm{o} 1))$ cuando $t_{2}-t_{1}$

$$
\rightarrow \infty \& \# \operatorname{Def}_{\mathrm{x}}=1
$$

Teniendo la definición de deferente, es fácil definir, en función de él, la órbita excéntrica y el epiciclo. La órbita excéntrica será aquella sobre la cual esté montada el deferente, en caso de estarlo sobre alguna órbita y el epiciclo será aquel que esté montado sobre el deferente o sobre otro epiciclo. Para evitar una definición circular (definiendo un epiciclo como aquél que puede estar montado sobre un epiciclo), diremos que el epiciclo está montado sobre una órbita que no es la excéntrica. En el caso de la órbita excéntrica, diremos que el conjunto puede tener un elemento o ninguno, pero no más de uno. En el caso de los epiciclos no podemos decir nada porque algunas órbitas no tienen (como el caso del Sol) y, si bien es cierto que en la teoría tal cual fue presentada por Ptolomeo había sólo un epiciclo por SOM, nada impide que se agreguen más (y de hecho, eso fueron haciendo a lo largo del tiempo).

\section{Definición auxiliar 4 (órbita excéntrica):}

(1) $o_{1} \in E x c_{x}$ syss $x \in P l \& o_{1} \in \mathbf{S O M}_{\mathbf{x}} \& \exists o_{2} \in \mathbf{S O M}_{\mathbf{x}}: o_{1}{ }^{m}{ }^{m} o_{2} \& o_{2} \in D e f_{x} \& \# E_{x c_{x}} \leq 1$

\section{Definición auxiliar 5 (epiciclo):}

(1) $o_{1} \in E p i_{x}$ syss $x \in P l, o_{1} \in \mathbf{S O M}_{x}, \exists o_{2} \in \mathrm{SOM}_{\mathrm{x}}: o_{2}{ }^{m} o_{1} \& o_{2} \notin E x c_{x}$

Luego podemos destacar, del conjunto de los puntos, al centro de la Tierra $\left(p_{T}\right)$ que será el punto que representa el centro de la Tierra, desde donde se realizan las observaciones (idealmente):

\section{Definición auxiliar 6 (centro de la Tierra):}

(1) $\Pi_{\mathrm{pT}}\left(\left\langle p_{a}, \ldots, p_{1}, \ldots, p_{z}\right\rangle\right)=p_{\mathrm{T}} \& p_{\mathrm{T}}$ es el centro de la Tierra.

La forma de definir un punto excéntrico es muy sencilla. Un deferente o una órbita excéntrica tiene punto excéntrico (los epiciclos no pueden tenerlo porque ellos giran sobre un punto móvil) si el centro de la órbita no es el centro de la Tierra. Habrá que exigir, entonces, dos cosas: que el centro de la órbita no se mueva y que no sea el centro de la Tierra. Que no se mueva lo expresaremos diciendo que los tres valores de la imagen de las coordenadas polares de ese punto se mantienen constantes. 


\section{Definición auxiliar 7 (punto excéntrico):}

(1) pex $_{0}={ }_{\text {def }} p_{1}$ syss $o \in E x c \cup \operatorname{Def}, p_{1}=c_{(0, t)} \neq p_{T} \&$ para todo $t \in T: \exists p_{2}, p_{3}, p_{4}, p_{5} \in P \& \exists ! h \in \mathbb{R}_{(0-360]}$ $\& \exists ! g \in \mathbb{R}_{(0-360]} \& \exists ! s \in \mathbb{R}^{+}$tal que co $\left(p_{1}, p_{2}, p_{3}, p_{4}, p_{5}, t\right)=(s, g, h)$

La definición de ecuante tampoco presenta dificultades. Una velocidad angular $(\omega)$ utiliza un ecuante cuando el centro de medición de la velocidad angular es distinto del centro de la órbita y la velocidad angular es constante para todo tiempo, es decir:

\section{Definición auxiliar 8 (punto ecuante):}

(1) peq $_{o}={ }_{\text {def }} p_{1}$ syss para toda $o \in O \& t \in T \& p_{1} \in P \& x \in \mathbb{R}_{(0-360):}\left(\omega\left(o, p_{1}, t\right)=x \& p_{1} \neq c_{(0, t)}\right.$

Al nuevo concepto que surja de la metrización de $\sigma$ lo llamaremos $S$ y será medido en partes. Nótese que partes es una unidad de medida relativa a cada planeta y, entonces, por ahora no permite establecer comparaciones entre los $S$ de distintos planetas. Se podrá decir que el radio de un deferente es tanto mayor que el de un epiciclo, pero dentro del mismo SOM. Sin embargo no es posible comparar, por ejemplo, los radios de los deferentes de dos SOM distintos. Nótese, por lo tanto, que aún cuando se convierte en un concepto métrico (porque los números ahora sí tendrán sentido matemático), sigue manteniendo, de todos modos, la característica que tenía $\sigma$ de no permitir comparaciones entre planetas. ${ }^{10}$ Por lo tanto:

\section{Definición auxiliar 9 (metrización de $\sigma$, primer paso):}

(1) $S: P \times P \times P l \times T \rightarrow \mathbb{R}^{+}$

(2) para todo $p l \in P l \& p_{1}, p_{2} \in P \& t_{1}, t_{2} \in T$ :

(a) $\sigma\left(p_{1}, t_{1}, t_{2}\right)=1 \rightarrow S\left(p_{1}, p_{T}, p l, t_{1}\right)<S\left(p_{1}, p_{T}, p l, t_{2}\right)$

(b) $\sigma\left(p_{1}, t_{1}, t_{2}\right)=-1 \rightarrow S\left(p_{1}, p_{T}, p l, t_{1}\right)>S\left(p_{1}, p_{T}, p l, t_{2}\right)$

Ello nos permite modificar el punto (c) de la ley fundamental, pidiendo ahora que si el brillo cambia, entonces cambie $S$; por supuesto, no exigiremos que si $S$ cambia, cambie también el brillo ni que si el brillo no cambia no cambie tampoco $S$ porque, nuevamente, puede haber cambios de distancia que no se vean reflejados en cambios de brillo.

\section{Reformulación del punto c (relativo al brillo) de la ley fundamental:}

(c') Si $b r\left(y, t_{1}, t_{2}\right)=1$, entonces $\left.S\left(p m_{\text {som(y) }}, p_{T}, y, t_{1}\right)<S\left(p m_{\text {som(y) }}\right), p_{T}, y, t_{2}\right) \&$ Si $b r\left(y, t_{1}, t_{2}\right)=-1$, entonces $S\left(p m_{\text {som }(y), p}, p_{T}, y, t_{1}\right)>S\left(p m_{\text {som(y) }}, p_{T}, y, t_{2}\right)$

Las condiciones establecen que si el $\sigma$ de un punto cambia, entonces también debe hacerlo el $\mathrm{S}$ medido desde el centro de la Tierra. No hemos puesto como condición que si $\sigma$ no cambia, no cambie tampoco la $S$ porque es posible que cambios en $S$ sean tan pequeños que no constituyan cambios visibles en el brillo. Por la misma razón no hemos exigido que cambios en $S$ impliquen cambios en $\sigma$.

$S$ nos dará la distancia en partes (relativas al planeta $P l$ ) del punto $p$ medido (el primero) desde otro punto que es, general pero no necesariamente, el centro de la Tierra $\left(p_{t}\right)$, en el tiempo $t$.

Habiendo ya metrizado $\sigma$, podemos definir el apogeo y el perigeo, como los instantes de tiempo en los que se alcanza la mayor y menor $S$, respectivamente, y las distancias relativas máximas y mínimas como las $S$ que se alcanzan en esos instantes.

\footnotetext{
${ }^{10}$ Medir en partes es como medir la altura de una persona utilizando como unidad de medida, por ejemplo, el largo de su dedo índice. Tiene sentido decir que una persona mide 25 dedos indices y que otra mide, en cambio, 28 dedos índices pero ello no permite decir que una es más alta que la otra. Son unidades de medidas relativas, en este caso, a cada individuo. Ptolomeo, en los sistemas de epiciclos y deferentes podía calcular sin dificultad las proporciones de las órbitas, es decir, medirlas tomando como unidad de medida a alguna de las órbitas en particular (por lo general, el deferente era igual a 60 partes -la definición de $S$ en función del radio del deferente puede verse en el apéndice 2). Ello era suficiente para poder predecir la longitud y latitud celeste del punto móvil del SOM de cualquier planeta. En efecto, no hacía falta conocer las distancias absolutas para que un SOM fuera un modelo de la teoría. Y, de hecho, en el Almagesto sólo calculó las distancias absolutas de la Luna y el Sol, que las necesitaba para los eclipses, pero la del resto de los planetas no las calcula.
} 


\section{Definición auxiliar 10 (apogeo):}

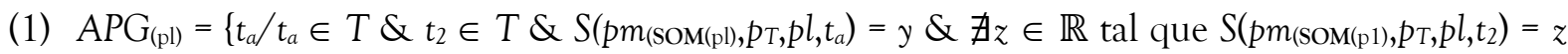
$\& z>y\}$

\section{Definición auxiliar 11 (perigeo):}

(1) $P E G_{(\mathrm{pl})}=\left\{t_{\mathrm{p}} / t_{p} \in T \& t_{2} \in T \& S\left(p m_{(\mathrm{SOM}(\mathrm{pl})}, p_{T}, p l, t_{\mathrm{p}}\right)=y \& \nexists z \in \mathbb{R}\right.$ tal que $S\left(p m_{(\mathrm{SOM}(\mathrm{p} 1)}, p_{T}, p l, t_{2}\right)=z$ $\& z<y\}$

\section{Definición auxiliar 12 (distancia máxima relativa):}

(1) $\operatorname{Smáx}_{(\mathrm{pl})}=_{\text {def }} m$ syss $t_{1} \in A P G_{(\mathrm{pl})} \& S\left(p m\left(\mathrm{SOM}_{(\mathrm{p} 1)}\right), p_{T}, p l, \mathrm{t}_{1}\right)=m$

\section{Definición auxiliar 13 (distancia mínima relativa):}

(1) $\operatorname{Smin}_{(\mathrm{pl})}=_{\text {def }} n$ syss $t_{1} \in P E G_{(\mathrm{pl})} \& S\left(p m_{(\mathrm{SOM}(\mathrm{p} 1)}, \mathrm{p}_{\mathrm{T}}, \mathrm{pl}, \mathrm{t}_{1}\right)=n$

Pero ahora debemos decir cómo se calcula la distancia en el apogeo y el perigeo, es decir, el método de determinación de las distancias máxima y mínima. La distancia en el apogeo es la sumatoria de todos los radios de las órbitas de un SOM más la distancia del centro de la Tierra a la excéntrica, si ésta existiera.

Definición auxiliar 14 (método de determinación de la distancia máxima relativa):

(1) para todo $\operatorname{SOM}_{(\mathrm{pl}):} \operatorname{Smáx}(\mathrm{pl})=S\left(p \operatorname{pex}_{\text {def }}, p \mathrm{~T}, p l, t\right)+\sum S\left(c_{(0, \mathrm{t})}, p m_{(o, t)}, p l, t\right)$

La distancia mínima, en cambio, es igual al radio del deferente menos la sumatoria de todas las otras órbitas y, de existir, también la distancia del punto excéntrico. ${ }^{11}$

Definición auxiliar 15 (método de determinación de la distancia mínima relativa):

(1) para todo $\operatorname{SOM}_{(\mathrm{pl}) \text { : }}$

(a) si $\exists o_{1} \in E x c_{p l}$, entonces $\left.S \min (\mathrm{pl})\right) S\left(c_{(\operatorname{def}(\mathrm{p}), t)}, p m_{(\operatorname{def}(\mathrm{pl}), t)}, p l, t\right)-\left[S\left(c_{(\operatorname{exc}(\mathrm{pl}), t)}, p_{T}, p l, t\right)+S\left(c_{(\operatorname{exc}(\mathrm{pl}), t)}\right)\right.$ $\left.\left.p m_{(\operatorname{exc}(p)), t)}, p l, t\right)+\sum S\left(c_{(\text {epi }(p)), t)}, p m_{(\text {epi }(p l), t)}, p l, t\right)\right]$

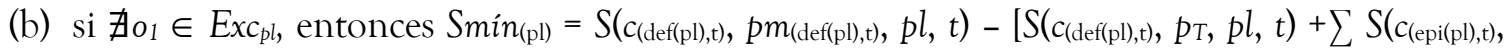

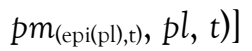

Puesto que tanto la distancia mínima como la máxima dan por resultado números positivos, la forma de calcular el perigeo que hemos dado supone que siempre el $S$ del radio del deferente es mayor que la suma de los $S$ de todas las otras órbitas que formen parte del SOM. Ello lo expresamos diciendo: ${ }^{12}$

Definición auxiliar 16 (el radio del deferente es mayor que la sumatoria de todos los otros):

(1) para todo $\operatorname{SOM}_{(\mathrm{pl})} \& t \in T, p l \in P l: S\left(\mathcal{c}_{(\operatorname{deff}(\mathrm{pl}), t)}, p m_{(\operatorname{def}(p)), t)}, p l, t\right)>\left[S\left(c_{(\operatorname{exc}(p)), t)}, p m_{(\operatorname{exc}(p)), t)}, p l, t\right)+\right.$ $S\left(\right.$ pex $\left.\left.\left._{\text {def }}, p_{T}, p l, t\right)+\sum S\left(c_{(\text {epi }}(p), t\right), p m_{(\text {epi }(p)), t)}, p l, t\right)\right]$

Podemos definir, además, un nuevo valor que determinará el grosor proporcional de un SOM y será la razón entre la distancia máxima y mínima relativas. De las definiciones de apogeo y perigeo podemos concluir que el apogeo será siempre mayor que el perigeo y, por lo tanto, la proporción entre ambos siempre será mayor que uno.

\footnotetext{
${ }^{11}$ Esto se da en todos los planetas excepto en Mercurio, que no tiene el perigeo a $180^{\circ}$ de su apogeo. Pero introducir esta diferencia complicaría considerablemente el sistema.

${ }^{12}$ Recuérdese que ésta era la otra característica esencial de un deferente, que aquí aparece como un corolario: no sólo es la órbita de mayor radio, sino que su radio es mayor a la suma de todas las otras órbitas. Ello impide que las órbitas, consideradas esferas cristalinas, choquen con la Tierra.
} 


\section{Definición auxiliar 17 (grosor relativo de un SOM):}

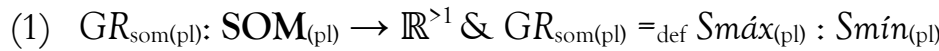

Como hemos dicho, la condición de ligadura que queremos expresar establece relaciones entre distintos SOM, por lo que necesitamos distancias absolutas, esto es, no expresadas en una unidad relativa a cada planeta (partes), sino en una común a todas (radios terrestres). Convertiremos, por lo tanto a $S$ en una distancia absoluta $(\hat{S})$, y será el último paso en la metrización de $\sigma . \hat{S}$, entonces, será una función igual que $S$, pero ya no será relativa a cada planeta (no los tendrá, por lo tanto, en su dominio) y, además, el valor que dé como resultado debe leerse como expresando radios terrestres. La relación entre $S$ y $\hat{S}$ es muy sencilla. El $\hat{S}$ de de un punto respecto de otro punto en un tiempo determinado es igual al $S$ de ese primer punto respecto de ese otro punto en el mismo tiempo, medido en las partes de un planeta determinado multiplicado por un $K_{p l}$ (que permanece constante para cada planeta y será llamado factor de absolutización para ese planeta). Como se ve, del dominio de $\hat{S}$ desaparece ya pl, porque la unidad de medida ya no depende más de cada planeta. ${ }^{13}$

\section{Definición auxiliar 18 (metrización de $\sigma$, segundo paso):}

(1) $\hat{S}: P \times P \times T \rightarrow \mathbb{R}^{+}$

(2) Condiciones (en relación con $S$ ): para todo $p l \in P l \& p_{1}, p_{2} \in P \& t \in T, \exists ! k_{p l} \in \mathbb{R}^{+}:$ $S\left(p_{1}, p_{T}, p l, t\right) \cdot K_{p l}=\hat{S}\left(p_{1}, p_{T}, t\right)$

Ahora podemos definir la distancia máxima y mínima absolutas y no ya relativas de cada planeta. Éstas podrían ser definidas de dos maneras, o bien diciendo que es la $\hat{S}$ que el planeta alcanza en sus apogeos y perigeos, o bien multiplicando la Smáx y Smín por el factor de absolutización de cada planeta. Lo haremos de la primera manera.

\section{Definición auxiliar 19 (distancia máxima absoluta):}

(1) $\hat{S} m a ́ x(\mathrm{pl})={ }_{\text {def }} m$ syss $t_{1} \in A P G_{(\mathrm{pl})} \& \hat{S}\left(p_{m}\left(\mathrm{SOM}(\mathrm{p} 1), p_{\mathrm{T}}, \mathrm{t}_{1}\right)=m\right.$

Definición auxiliar 20 (distancia mínima absoluta):

(1) $\hat{S}_{\min }(\mathrm{pl})={ }_{\text {def }} n$ syss $t_{1} \in P E G_{(\mathrm{pl})} \& \hat{S}\left(p_{(\mathrm{SOM}(\mathrm{p} 1)}, p_{T}, t_{1}\right)=n$

También es posible definir el grosor absoluto de un determinado SOM, análogamente a como lo hicimos con el relativo.

\section{Definición auxiliar 21 (grosor absoluto de un SOM):}

(1) $\mathrm{GA}_{\mathrm{som}(\mathrm{pl}):} \operatorname{SOM}_{(\mathrm{pl})} \rightarrow \mathbb{R}^{>1} \& \mathrm{GR}_{\mathrm{som}(\mathrm{pl})}={ }_{\mathrm{def}} \hat{S}_{\operatorname{má}}(\mathrm{pl}): \hat{S}_{m i n}(\mathrm{pl})$

De la definición que hemos dado de $\hat{S}$ en función de $\mathrm{S}$, se sigue, como corolario, que los grosores absolutos y relativos son iguales. La demostración puede verse en el apéndice 3 .

Ahora debemos definir el orden de los planetas. Será una función ordenadora (ord) que otorgue a cada planeta un número natural, de tal manera que el que tenga el número menor, estará más cerca de la Tierra.

\section{Definición auxiliar 22 (función ordenadora de los planetas):}

(1) ord: $P l \rightarrow \mathbb{N}$

Los criterios para atribuir estos valores son variados y complejos. En el caso de los planetas exteriores,

\footnotetext{
${ }^{13}$ Como las distancias absolutas no son más que las relativas multiplicadas por un factor de absolutización, evidentemente un cambio en la relativa levará a uno proporcional en la absoluta, de tal manera que reescribir el punto (c) de la ley fundamental en función de las distancias absolutas no agrega nada. Además, no sería adecuado porque mientras el sistema le permitía sin dificultad obtener las distancias relativas, no era para nada fácil obtener las absolutas.
} 
podría ser el período de revolución del deferente. A mayor período, mayor distancia. En el caso de los planetas interiores tiene que ver con la complejidad del movimiento, a mayor complejidad, menor distancia. La complejidad podría expresarse en función de la cantidad de órbitas (cuantas más órbitas, más complejo el sistema). Estos criterios pueden aplicarse de la siguiente manera: si utilizamos en primer lugar el criterio del período de revolución a los siete planetas, el que tiene mayor período de revolución es la Luna, por lo que la Luna sería el planeta más cercano. Luego vendrían el Sol, Mercurio y Venus, que tienen igual período, menor que el de la Luna, pero mayor al del resto de los planetas, por lo que sabremos que ellos ocupan el segundo, tercer y cuarto puesto, y luego continuamos ubicando a los planetas exteriores, primero Marte, que tendrá la ubicación quinta, luego Júpiter sexto- y finalmente Saturno -séptimo-. Necesitamos, entonces, un criterio para discriminar entre los que "empatan" respecto del primer criterio y ese puede ser, perfectamente, el de mayor complejidad. Mercurio tiene más órbitas que Venus y Venus que el Sol. Por lo tanto, con estos dos criterios pueden ordenarse los siete planetas:

\section{Definición auxiliar 23 (criterio de determinación de la función ordenadora de planetas):}

(1) $\operatorname{si} t \in T \& n, m \in \mathbb{N} \& x, z \in P l \& \operatorname{ord}_{(\mathrm{x})}=n \& \operatorname{ord}_{(z)}=m, \&\left(\mathbf{S O M}_{\mathbf{x}}, \mathbf{S O M}_{z} \in \mathbf{M}(\mathbf{S E D}), \rightarrow\right.$

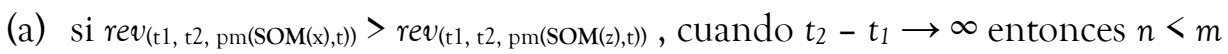

(b) si $\operatorname{rev}_{(\mathrm{t} 1, \mathrm{t} 2, \mathrm{pm}(\operatorname{SOM}(\mathrm{x}), \mathrm{t}))}<\operatorname{rev}(\mathrm{t} 1, \mathrm{t} 2, \mathrm{pm}(\operatorname{SOM}(z), t))$, cuando $t_{2}-t_{1} \rightarrow \infty$ entonces $n>m$

(c) si $\operatorname{rev}(\mathrm{t} 1, \mathrm{t} 2, \mathrm{pm}(\operatorname{SOM}(\mathrm{x}), \mathrm{t}))=\operatorname{rev}\left(\mathrm{t} 1, \mathrm{t} 2, \mathrm{pm}(\mathrm{SOM}(z), \mathrm{t})\right.$, cuando $t_{2}-t_{1} \rightarrow \infty \& \# \mathrm{O} \in \mathrm{SOM}_{\mathrm{x}}<\# \mathrm{O} \in \mathrm{SOM}_{z}$ entonces $n>m$

(d) $\sin \operatorname{rev}_{(\mathrm{t} 1, \mathrm{t} 2, \mathrm{pm}(\mathrm{SOM}(\mathrm{x}, \mathrm{t}))}=\operatorname{rev}_{(\mathrm{t} 1, \mathrm{t} 2, \mathrm{pm}(\operatorname{SOM}(z), \mathrm{t}))}$, cuando $t_{2}-t_{1} \rightarrow \infty \& \# \mathrm{O} \in \mathrm{SOM}_{\mathrm{x}}>\# \mathrm{O} \in \mathrm{SOM}_{z}$ entonces $n<m$

Estos requisitos bastan para ordenar a todos los planetas. La aplicación de la función da los siguientes $\operatorname{resultados:}_{\operatorname{ord}_{(\mathrm{Lu})}}=1, \operatorname{ord}_{(\mathrm{Me})}=2, \operatorname{ord}_{(\mathrm{Ve})}=3, \operatorname{ord}_{(\mathrm{Sol})}=4, \operatorname{ord}_{(\mathrm{Ma})}=5, \operatorname{ord}_{(\mathrm{Ju})}=6, \operatorname{ord}_{(\mathrm{Sa})}=7$.

\subsubsection{Condición de ligadura de las distancias máximas y mínimas de los planetas}

Finalmente ya tenemos todos los elementos para poder expresar la condición de ligadura. Ésta dice que, dados dos planetas, uno inmediatamente superior que el otro, la distancia máxima absoluta $\left(\hat{S}_{\text {max }}\right)$ del menor tiene que ser igual a la distancia mínima absoluta $\left(\hat{S}_{\text {mín }}\right)$ del mayor:

\section{Definición 4:}

$\mathrm{C}(\mathrm{SED}): \chi \in \mathrm{C}(\mathrm{SED})$ syss $\varnothing \neq \chi \subset \mathrm{M}(\mathrm{SED})$ y para todo $x, x^{\prime} \in X$ :

$$
\text { Si } y, z \in P l_{y} \cap P l_{z} \& \operatorname{ord}(z)=\operatorname{ord}(y)+1 \text {, entonces } \hat{S}_{\max _{(y)}}=\hat{S} \min (z)_{1}
$$

La condición de ligadura, en realidad, no constituye ninguna restricción en sentido fuerte. Es decir, del SOM de cada planeta lo único que podemos obtener son las distancias relativas y, por lo tanto, también el $G R_{\text {som }}$ de ese sistema. La condición de ligadura exige que, en dos planetas contiguos, la distancia máxima absoluta del más cercano sea igual a la mínima absoluta del más alejado. Pero el único vínculo que tenemos entre las distancias máximas y mínimas absolutas por un lado y las relativas por otro, es la igualdad entre sus proporciones $\left(G R_{\text {som }}=G_{\text {som }}\right)$. Con esos datos no es posible conocer ninguna distancia absoluta (porque, en realidad, nos falta un factor de absolutización $\left(K_{p l}\right)$ ).

\subsubsection{Vínculos interteóricos}

$\mathrm{Si}$, en cambio, existiera algún vínculo interteórico que nos proveyera de alguna distancia absoluta o de algún factor de absolutización, podríamos, a través de los datos que tenemos, calcular todos los demás. Y en efecto, existen tales vínculos. 


\subsubsection{Vínculo interteórico para obtener la distancia máxima de la Luna}

A través de un vínculo interteórico con la Teoría de la Distancia Absoluta de la Luna (DAL) que calcula la distancia máxima y mínima absolutas de la Luna mediante la paralaje (H408-H416), puedo

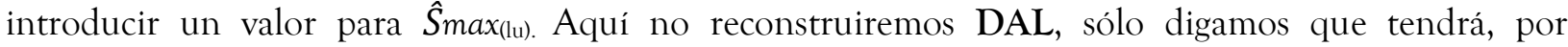
supuesto, $P, P l$, etc. y $\hat{S}_{m a ́ x}\left(\mathrm{pl)}\right.$ y $\hat{S}_{\min }(\mathrm{pl)})$. El vínculo interteórico diría:

\section{Definición 5:}

$\left.\mathrm{L}_{1}(\mathrm{SED}) \subseteq \mathrm{M}(\mathrm{DAL}) \times \pi(\mathrm{DAL},[\mathrm{Lu}, \hat{\mathrm{S}} \operatorname{máx}(\mathrm{p})])\right) \times \mathrm{M}_{\mathrm{p}}(\mathrm{SED}) \times \pi(\mathrm{SED},[\mathrm{Lu}, \hat{S} \operatorname{máx}(\mathrm{pl})])$ tal que:

(1) Para todo $x \in \mathbf{M}(\mathrm{DAL}) \&$ para todo $z \in \mathbf{M}_{\mathrm{p}}(\mathrm{SED})$ :

$$
\left\langle x,\left\langle L_{x}, \hat{S}_{\max (\mathrm{lu}) \times}\right\rangle, z,\left\langle\operatorname{Lu}_{z}, \hat{S} \max _{(\mathrm{lu})_{z}}\right\rangle\right\rangle \in \mathrm{L}_{1}(\mathrm{SED}) \text { syss } \hat{S}_{\max } \operatorname{mlu}_{\mathrm{x}}=\hat{S}_{\max } \operatorname{ma}_{(\mathrm{lu})_{z}}
$$

Y con ese dato obtenido a través de la condición de ligadura C(SED) ya es posible establecer todos los valores de distancias máxima y mínima absolutas ya que, conociendo una, es posible conocer todas las demás (véase un ejemplo en el apéndice 4).

\subsubsection{Vínculo interteórico para obtener la distancia mínima del Sol}

A través de un cálculo utilizando un diagrama de eclipses lunares y solares y teniendo en cuenta el valor obtenido para la distancia absoluta de la Luna, Ptolomeo es capaz de calcular la distancia absoluta del Sol (H422-H425). Aquí tampoco lo reconstruiremos, pero lo llamaremos DAS. Ello nos permite establecer un nuevo vínculo interteórico.

\section{Definición 6:}

$\mathrm{L}_{2}(\mathrm{SED}) \subseteq \mathrm{M}(\mathrm{DAL}) \times \pi(\mathrm{DAL},[\mathrm{Sol}]) \times \mathbf{M}_{\mathrm{p}}(\mathrm{SED}) \times \pi\left(\mathrm{SED},\left[\right.\right.$ Sol, $\left.\left.\hat{S}_{\text {máx }}(\mathrm{pl})\right]\right)$ tal que:

(1) Para todo $x \in \mathbf{M}(\mathrm{DAS}) \&$ para todo $z \in \mathrm{M}_{\mathrm{p}}(\mathrm{SED})$ :

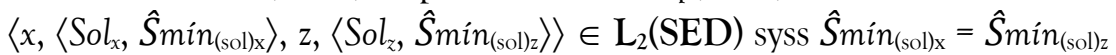

\subsection{Las aplicaciones propuestas y el elemento teórico básico del Sistema de Epiciclos}

\section{y Deferentes de Ptolomeo}

El dominio de aplicaciones propuestas I constituye la clase de aquellos sistemas empíricos a los que uno desea aplicar la ley fundamental de la teoría. Ellos no pueden ser caracterizados por medios puramente formales. Lo único que podemos decir desde un punto de vista formal es que una aplicación propuesta es un modelo parcial. Aquí, esto significa que $\mathbf{I}(\mathrm{SED}) \subseteq \mathbf{M}_{\mathrm{pp}}(\mathrm{SED})$ y que los miembros de $\mathbf{I}(\mathrm{SED})$ son sistemas empíricos compuestos por planetas, es decir por estrellas errantes, que no respetan la distancia relativa entre sí con longitudes y latitudes observadas a lo largo del tiempo. Como hemos ya dicho, en la época de Ptolomeo habían identificado siete.

El elemento teórico básico del Sistema de Epiciclos y Deferentes de Ptolomeo puede ahora ser caracterizado como T(SED): $=\langle\mathrm{K}(\mathrm{SED}), \mathrm{I}(\mathrm{SED})\rangle$.

\subsection{La aserción empírica del Sistema de Epiciclos y Deferentes de Ptolomeo}

SED pretende que ciertos sistemas empíricos, descritos SED-no-teóricamente, satisfacen las condiciones impuestas por él en el siguiente sentido: esos son los datos de la experiencia que se deberían obtener, si la realidad se comporta como ella dice. Esta pretensión se expresa en la aserción empirica de SED, que puede formularse de la siguiente manera:

$\left({ }^{*}\right) \quad$ Todo planeta puede ser, añadiendo un conjunto de componentes teóricos ${ }^{m}, S_{\text {SOM y }} p m_{\text {som }}$ a la parte no-teórica del núcleo teórico correspondiente $(\langle P, P l, T, O, \mathbb{R}, \mathbb{N}, p u n, c o, r, c, \omega, p m, \lambda, \beta$, $\sigma, b r\rangle)$, aproximadamente extendido a un modelo del Sistema de Epiciclos y Deferentes de Ptolomeo. 
El núcleo teórico presentado sirve como núcleo teórico básico para todas las aplicaciones propuestas de SED. Lo que, en concreto, afirma la aserción empírica es que para cada planeta es posible construir teóricamente un sistema de epiciclos y deferentes tal que la longitud y latitud celeste de su punto móvil coincida con la longitud y latitud celeste observada del planeta y, además, que el brillo del planeta observado sea inversamente proporcional a la distancia entre la Tierra y el punto móvil del SOM en cualquier instante de tiempo.

\section{Las especializaciones y la red teórica del Sistema de Epiciclos y Deferentes de Ptolomeo}

En el sistema de Ptolomeo hay varias leyes especiales. En primer lugar hay dos (que tienen una versión fuerte y una débil) que se predican de los planetas interiores y de los planetas exteriores. De estas leyes queda excluido, evidentemente, el Sol que tendrá dos leyes especiales propias y la Luna (que tiene sus leyes, pero aquí no las reconstruiremos). ${ }^{14}$ Ambos, el Sol y la Luna, a diferencia de los planetas interiores y exteriores, no tienen retrogradaciones. La mejor forma de distinguirlos es, primero, definir retrogradación, y luego decir que esas leyes especiales se aplican sólo a los que retrogradan. Definamos, entonces retrogradación.

Intuitivamente la retrogradación se produce cuando un planeta, en vez de avanzar sobre el cielo, retrocede. Ello quiere decir que la retrogradación podría definirse para un planeta determinado como el subconjunto de instantes de tiempo en los que la longitud retrocede. Que la función longitud retrocede podemos decirlo simplemente afirmando que su derivada es negativa. Recordemos que ya habíamos exigido que la función longitud fuera continua (parcialmente) en todos sus valores excepto en el 0 y el 360.

\section{Definición auxiliar 24 (instantes de retrogradación): ${ }^{15}$}

(1) $\operatorname{Ret}_{(\mathrm{p} 1)}=\{t / t \in T \& p l \in P l \& D \lambda(p l, t)<0\}$

Ahora ya podemos distinguir a los planetas que retrogradan de aquellos que no lo hacen.

\subsection{Leyes especiales para los planetas que retrogradan}

El sistema de Ptolomeo distinguía los planetas interiores de los exteriores. Hay varios criterios para hacerlo, pero probablemente el mejor sea que los exteriores aparecen a veces en oposición al Sol, mientras que los interiores nunca lo hacen. En una versión débil, lo que dicen las leyes especiales es: si un planeta es exterior, el período de revoluciones del epiciclo ${ }^{16}$ es igual al período de revoluciones del Sol. En un planeta interior, en cambio, la igualdad se da con el período de revoluciones del deferente.

\subsubsection{Versión débil de las leyes especiales para los planetas que retrogradan}

\subsubsection{Ley especial para Planetas Interiores}

\section{Definición 7:}

M(IDSED): $x$ es un sistema de epiciclos y deferente de Ptolomeo de planeta interior (débil) $(x \in \mathbf{M}(\mathrm{IDSED}))$ syss

\footnotetext{
${ }^{14}$ Por ejemplo, una que vincule las fases de la luna con su elongación.

${ }^{15}$ Con esta definición de retrogradación se nos escapa el instante en el que un planeta está retrogradando y justo tiene longitud 0 . Para incluirlo bastaría decir simplemente que si el instante posterior y anterior pertenecen al conjunto de las retrogradaciones, entonces ese instante también pertenecerá.

Definición auxiliar 24': (Inclusión del instante de longitud 0 en la retrogradación)

(1) $\operatorname{Si} t_{1}, t_{2}, t_{3} \in T \& t_{1}, t_{3} \in R e t_{(p))} \& t_{1}<t_{2}<t_{3} \& \nexists t_{4}, t_{5} \in T \& \notin \operatorname{Ret}_{(p))}$ tal que $t_{1}<t_{4}<t_{2}<t_{5}<t_{3}$, entonces $t_{2} \in \operatorname{Ret}_{(\mathrm{pl})}$

${ }^{16}$ Si hubiera más de uno, se cumpliría con el primero pero como en el sistema de Ptolomeo no aparece nunca más de un epiciclo, omitiremos esta precisión.
} 
(1) $x \in \mathrm{M}(\mathrm{SED})$

(2) para todo $t_{1}, t_{2} \in T \& y \in P l$

Si $\operatorname{pun}(\operatorname{Sol})=s \& \operatorname{pun}(y)=n \& \exists \mathrm{t}_{x} \in \operatorname{Ret}_{(y)} \&$ para todo $t:|\lambda(s, t)-\lambda(n, t)|<180$, entonces:

$\operatorname{rev}_{(\mathrm{t} 1, \mathrm{t} 2, \operatorname{pm}(\operatorname{SOM}(\mathrm{sol}), \mathrm{t}))}=\operatorname{rev}_{(\mathrm{t} 1, \mathrm{t} 2, \operatorname{pm}(\operatorname{def}(x), \mathrm{t}))}$

Como es notación habitual, $|x|$ significa el valor absoluto de $x$. Esta ley especial afirma, entonces, que si existe un tiempo en el que el planeta retrograda -lo que excluye al Sol y la Luna- y, además, el valor absoluto de la longitud del Sol menos la longitud del planeta -esto es, su elongación- siempre es menor que 180, es decir, nunca está en oposición al Sol -o sea, se trata de un planeta interior-, entonces la cantidad de revoluciones para cualquier tiempo del punto móvil del deferente del planeta es igual a la cantidad de revoluciones del punto móvil del SOM del Sol.

\subsubsection{Ley especial para Planetas Exteriores}

\section{Definición 8:}

M(EDSED): $x$ es un sistema de epiciclos y deferente de Ptolomeo de planeta exterior (débil) $(x \in \mathbf{M}(\mathrm{EDSED})$ ) syss

(1) $x \in \mathrm{M}(\mathrm{SED})$

(2) para todo $t_{1}, t_{2} \in T \& y \in P l$

$\operatorname{Si} \operatorname{pun}(\operatorname{Sol})=s \& \operatorname{pun}(y)=n \& \exists t_{x} \in \operatorname{Ret}_{(y)} \& \exists t_{z} \in T /\left|\lambda\left(s, t_{z}\right)-\lambda\left(n, t_{z}\right)\right|=180$, entonces:

$\operatorname{rev}_{(\mathrm{t} 1, \mathrm{t} 2, \mathrm{pm}(\mathrm{SOM}(\mathrm{sol}), \mathrm{t}))}=\operatorname{rev}_{(\mathrm{t} 1, \mathrm{t} 2, \mathrm{pm}(\mathrm{Epi}(\mathrm{x}), \mathrm{t}))}$

Lo sostiene esta ley es, por lo tanto, que si el planeta retrograda y, además, existe un tiempo en el cual el valor absoluto de la longitud del Sol menos la longitud del planeta es igual a 180, esto es, está en oposición al Sol -o sea, es un planeta exterior-, entonces las revoluciones para cualquier tiempo del punto móvil del epiciclo del planeta es igual a las del punto móvil del SOM del Sol.

\subsubsection{Versión fuerte de las leyes especiales para los planetas que retrogradan}

En realidad, las leyes especiales podrían decir un poco más. En el caso de los planetas exteriores podría decir no sólo que el período coincide sino que la línea que une al punto móvil del epiciclo con su centro es paralela a la línea que une al centro de la Tierra con el Mean Sun (Sol medio) -ya definiremos este término- y, además, ambas están en el mismo sentido (apuntan hacia el mismo lado). En el caso de los planetas interiores - pero esto se da sólo con Venus, porque Mercurio tiene una complicación mayor-, las líneas paralelas son, en el caso del Sol la misma, y en el planeta la que une el punto ecuante del deferente con el punto móvil de éste (véanse las figuras 3 y 4).
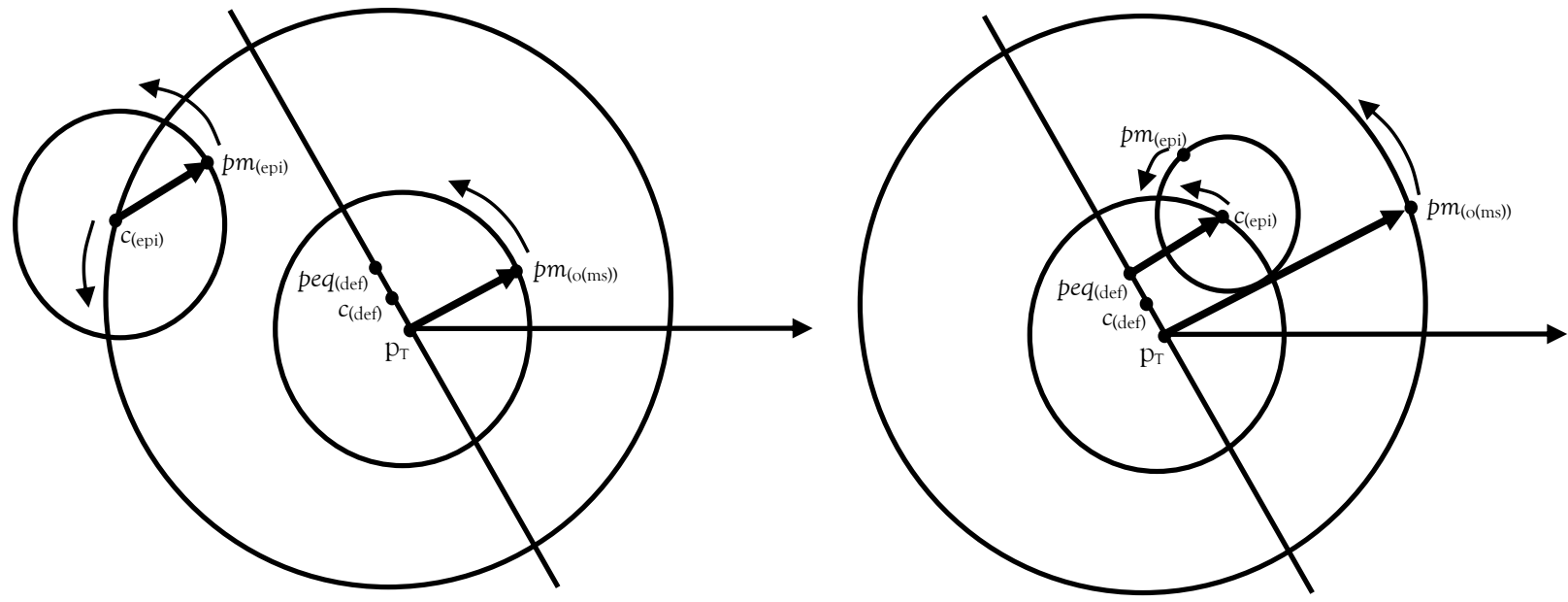

Figuras 3 y 4 . 
Antes de continuar, debemos precisar qué quiere decir sol medio. Es un concepto teórico introducido por Ptolomeo y consiste en el punto en el que se encontraría el Sol si su velocidad fuera constante. Si Ptolomeo hubiera decidido reconstruir el SOM del Sol mediante un epiciclo y un deferente centrado en la Tierra en vez de introducir el punto excéntrico, el sol medio coincidiría con el punto móvil del deferente. Como no lo ha hecho, debemos definir una nueva órbita sobre la que gire el sol medio. Esta órbita tiene la misma velocidad y el mismo radio que el deferente del Sol, pero está centrada en el centro de la Tierra, no en la excéntrica. Además, las longitudes de los puntos móviles de ambas órbitas coinciden en el apogeo y el perigeo del Sol (ver infra figura 6).

Definición auxiliar 25 (órbita del Sol medio):

(1) $\mathrm{O}_{(\mathrm{ms})}=\left\{0 / o \in O \& r_{(\mathrm{o})}=r_{(\mathrm{def}(\mathrm{sol}))} \& \omega_{(\mathrm{o}, \mathrm{pT}, \mathrm{t})}=\omega_{(\text {(def(sol),pex(deff(sol)),t) }} \& c_{(\mathrm{o})}=p_{\mathrm{T}} \&\right.$ para todo $t \in A P G_{(\mathrm{sol})} \cup$ $\left.P E G_{(\mathrm{sol}):} \lambda_{(\mathrm{pm}(\mathrm{o}), \mathrm{t})}=\lambda_{(\mathrm{pm}(\text { (epi(sol) }) \mathrm{t})}\right\}$

\subsubsection{Ley especial para Planetas Interiores}

Una última aclaración: para expresar las leyes en versión fuerte utilizaremos la noción de vector (vec), que no es necesario definir, la podemos tomar prestada de la matemática y lo determinaremos a partir de dos puntos, el primero expresará el origen y el segundo el final, de tal manera que quede así expresado también el sentido. Además, utilizaremos el símbolo $\uparrow$ para expresar igual dirección y sentido. Ahora sí, la versión fuerte de la ley especial de los planetas interiores diría que:

\section{Definición 9:}

M(IFSED): $x$ es un sistema de epiciclos y deferente de Ptolomeo de planeta interior (fuerte) $(x \in \mathbf{M}($ IFSED)) syss

(1) $x \in \mathrm{M}(\mathrm{SED})$

(2) para todo $t \in T \&$ para todo $y \in P l$ Si $\operatorname{pun}(\operatorname{Sol})=s \& \operatorname{pun}(y)=n \& \exists t_{x} \in \operatorname{Ret}_{(y)} \&$ para todo $t:|\lambda(s, t)-\lambda(n, t)|<180$, entonces: $\operatorname{vec}\left(p_{T}, p m_{(\mathrm{o}(\mathrm{ms}), \mathrm{t})}\right) \uparrow \uparrow \operatorname{vec}\left(\operatorname{peq}(\operatorname{Def}(\mathrm{x})), p m_{(\operatorname{Def}(\mathrm{x}, \mathrm{t})}\right)$

\subsubsection{Ley especial para Planetas Exteriores}

Y la versión fuerte de la ley especial de ley especial para los planetas exteriores diría:

\section{Definición 10:}

M(EFSED): $\quad x$ es un sistema de epiciclos y deferente de Ptolomeo de planeta exterior (fuerte) $(x \in \mathbf{M}(\mathrm{EFSED}))$ syss

(1) $x \in \mathrm{M}(\mathrm{SED})$

(2) para todo $t \in T \&$ para todo $y \in P l$

$\operatorname{Si} \operatorname{pun}(\operatorname{Sol})=s \& \operatorname{pun}(y)=n \& \exists t_{x} \in \operatorname{Ret}_{(y)} \& \exists t_{z} \in T /\left|\lambda\left(s, t_{z}\right)-\lambda\left(n, t_{z}\right)\right|=180$, entonces:

$\operatorname{vec}\left(p T, p m_{(o(m), t)}\right) \uparrow \uparrow \operatorname{vec}\left(c_{(E p i(x)),} p m_{(E p i(x), t)}\right)$

\subsubsection{Teoremas de las leyes especiales (versión fuerte)}

De las leyes especiales en su versión fuerte puede demostrarse -pero no lo haremos aquí- que, justo en la mitad de la retrogradación, la elongación del planeta, en el caso de los exteriores es igual a $180^{\circ} \mathrm{y}$, en el caso de los interiores, igual a $0^{\circ}$. Podemos, por lo tanto, formalizar estos teoremas de la siguiente manera.

\subsubsection{Definiciones auxiliares}

Primero debemos poder ubicar el instante medio de una retrogradación y, para ello, debemos individualizar cada retrogradación porque, hasta ahora, era sólo el conjunto de todos los instantes de tiempo en los que el planeta retrogradó. Para hacerlo, bastará definir los subconjuntos dentro de Ret(p) 
que reúna a los instantes de tiempo implicados en cada retrogradación. Todo elemento de Ret(p) pertenecerá a uno y sólo un subconjunto. Para identificar los instantes de tiempo que pertenecen a un subconjunto, diremos que si existe algún instante $t_{2}$ que no pertenece a $\operatorname{Ret}_{(\mathrm{p})}$ pero está ubicado entre dos instantes que sí pertenecen $\left(t_{1}\right.$ y $\left.t_{3}\right)$, entonces esos dos instantes pertenecen a distintos subconjuntos. Si no existe tal instante, pertenecen al mismo.

\section{Definición auxiliar 26 (identificación de cada retrogradación):}

(1) $\operatorname{ret}_{(\mathrm{p}, \mathrm{n})}=\left\{t_{n} / t_{n} \in \operatorname{Ret}_{(\mathrm{p} 1)} \& x, z \in \mathbb{N} \& t_{y} \in T \& t_{1}<t_{y}<t_{2} \&\right.$

(a) $t_{y} \in \operatorname{Ret}_{(\mathrm{pl})} \rightarrow t_{1}, t_{2}, t_{\mathrm{y}} \in \operatorname{ret}_{(\mathrm{p} 1, \mathrm{x})}$

(b) $\left.t_{\mathrm{y}} \notin \operatorname{Ret}_{(\mathrm{pl})} \rightarrow \mathrm{t}_{1} \in \operatorname{ret}_{(\mathrm{p} 1, \mathrm{x})} \& t_{1} \notin \operatorname{ret}_{(\mathrm{p} 1, z)} \& t_{2} \in \operatorname{ret}_{(\mathrm{p} 1, z)} \& t_{2} \notin \operatorname{ret}_{(\mathrm{p} 1, \mathrm{x})}\right\}$

Ahora identificaremos el instante medio de una retrogradación. El instante medio es, evidentemente el valor promedio del tiempo inicial y el final. Por lo tanto, debemos ubicar al tiempo inicial y final de una determinada retrogradación. Ello no es difícil, si un instante $t_{1}$ pertenece a una determinada retrogradación y no hay ningún instante anterior que pertenezca, será el instante inicial y lo contrario valdrá para el instante final. Identifiquemos, entonces, el tiempo medio de una retrogradación:

\section{Definición auxiliar 27 (instante medio de una retrogradación):}

(1) $t_{m(\operatorname{ret}(x, z))}={ }_{\text {def }} t_{m}$ tal que $x \in P l \& z \in \mathbb{N} \& t_{1}, t_{2}, t_{i}, t_{f}, t_{m} \in T \& t_{i} \in \operatorname{ret}_{(x, z)} y \nexists t_{1} \in \operatorname{ret}_{(x, z)}$ tal que $t_{1}<t_{i}$ $\& t_{f} \in \operatorname{ret}_{(\mathrm{x}, z)} \& \nexists t_{3} \in \operatorname{ret}_{(\mathrm{x}, \mathrm{z})}$ tal que $\left.t_{2}>t_{f} \& t_{m}=\left(t_{f}-t_{1}\right) / 2\right)$

Ahora sí podemos establecer los teoremas.

\subsubsection{Teorema de M(IFSED): La elongación en el medio de una retrogradación es $0^{\circ}$}

\section{Definición 11:}

(1) Si $x \in \mathbf{M}($ IFSED) $\&$ Sol $\in P l \&$ pun $(\operatorname{Sol} l)=s \& p u n(x)=n \& z \in \mathbb{N}$, entonces: $\lambda\left(s, t_{m(\operatorname{ret}(x, z))}\right)-\lambda\left(n, t_{m(\operatorname{ret}(x, z))}\right)=0$

\subsubsection{Teorema de M(EFSED): La elongación en el medio de una retrogradación es $180^{\circ}$}

\section{Definición 12:}

(1) Si $x \in \mathbf{M}($ IFSED $) \&$ Sol $\in P l \& p u n(\operatorname{Sol})=s \& \operatorname{pun}(x)=n \& z \in \mathbb{N}$, entonces: $\mid \lambda\left(s, t_{m(\operatorname{ret}(x, z))}-\lambda(n\right.$, $\left.t_{m(\operatorname{ret}(x, z))}\right) \mid=180$

\subsubsection{Leyes especiales que determinan la longitud del punto móvil del deferente en el instante medio de una retrogradación}

De estas dos leyes especiales derivadas se pueden extraer, a su vez, dos importantes leyes nuevas. La primera se relaciona con la ley de los planetas interiores y afirma que, en el instante medio de la retrogradación, cuando la longitud del Sol y la del planeta coinciden, también coincidirá la longitud del punto móvil del deferente del planeta siempre que en el instante del centro de la retrogradación estén alineados el centro de la Tierra, el centro del deferente, el centro del epiciclo y el punto móvil del epiciclo. Es un corolario importante porque permite determinar una posición real del punto móvil del deferente y así, ya puede conocerse siempre su ubicación. La segunda ley, relacionada con los planetas exteriores, dice que las longitudes del planeta y de su deferente se identificarán cuando estén en el punto medio de la retrogradación y ambas, por lo tanto, estarán a $180^{\circ}$ de la longitud del Sol siempre 
que todos los puntos antes mencionados estén alineados. Aquí si se trata de nuevas leyes especiales porque exigen más condiciones (la alineación de los puntos).

Para decir que los puntos que hemos mencionado están alineados en el instante medio de la retrogradación, utilizaremos pero no definiremos ${ }^{17}$ una relación de alineación (alin) que dirá, simplemente, que los puntos en ella incluidos están alineados en un instante $t$ determinado.

\subsubsection{Ley especial que determina la longitud del punto móvil del deferente} para planetas interiores o Ley de retrogradación de los planetas interiores

(IRSED)

\section{Definición 13:}

M(IRSED): $x$ es un sistema de epiciclos y deferente de Ptolomeo de retrogradación de planeta interior $(x \in$ M(IRSED)) syss

(1) $x \in \mathrm{M}(\mathrm{SED})$

(2) para todo $y \in P l$

Si Sol, $y \in P l \& \operatorname{pun}(\operatorname{Sol})=s \& \operatorname{pun}(y)=n \& z \in \mathbb{N} \& \exists t_{x} \in \operatorname{Ret}_{(y)} \&$ para todo $t:$

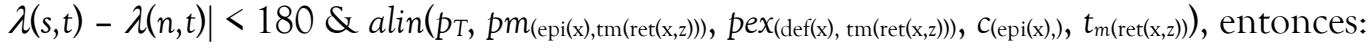

$\lambda\left(\operatorname{pm}\left(\operatorname{def} f_{x}, t_{\mathrm{m}(\operatorname{ret}(x, z))}\right), \mathrm{t}_{\mathrm{m}(\operatorname{ret}(\mathrm{x}, z)))}\right) \lambda\left(n, t_{m(\operatorname{ret}(x, z))}\right)=\lambda\left(s, t_{m(\operatorname{ret}(x, z))}\right)$

2.1.4.2. Ley especial que determina la longitud del punto móvil del deferente para planetas Exteriores o Ley de retrogradación de los planetas Exteriores (ERSED)

\section{Definición 14:}

M(ERSED): $x$ es un sistema de epiciclos $y$ deferente de Ptolomeo de retrogradación de planeta exterior $(x \in$ $\mathrm{M}(\mathrm{ERSED}))$ syss

(1) $x \in \mathrm{M}(\mathrm{SED})$

(2) para todo $y \in P l$

Si Sol $\in P l \& \operatorname{pun}(\operatorname{Sol})=s \& \operatorname{pun}(y)=n \& z \in \mathbb{N} \& \exists t_{x} \in \operatorname{Ret}_{(y)} \& \exists t_{z} \in T /\left|\lambda\left(s, t_{z}\right)-\lambda\left(n, t_{z}\right)\right|=180$

$\& \operatorname{alin}\left(p_{T}, p m_{(\operatorname{epi}(x), \operatorname{tm}(\operatorname{ret}(x, z))),} \operatorname{pex}(\operatorname{def}(x), \operatorname{tm}(\operatorname{ret}(x, z))), c_{(\operatorname{epi}(x),),} t_{m(\operatorname{ret}(x, z)))}\right.$, entonces:

$\lambda\left(p m\left(\operatorname{def} f_{x}, t_{m(\operatorname{ret}(x, z))}\right), t_{m(\operatorname{ret}(x, z))}\right)=\lambda\left(n, t_{m(\operatorname{ret}(x, z))}\right)=\lambda\left(s, t_{m(\operatorname{ret}(x, z))}\right) \pm 180$

\subsection{Leyes especiales para el Sol}

\subsubsection{Ley especial para el Sol respecto de la Latitud Celeste}

Una ley que cumple el Sol (aunque es analítica) pide que su latitud celeste siempre sea 0 . Esto porque la latitud es el ángulo que separa un punto del plano de la eclíptica pero el plano de la eclíptica es el plano de la órbita solar. ${ }^{18}$ Por lo tanto:

\section{Definición 15:}

M(S $\beta$ SED): $x$ es un sistema de epiciclos y deferente de Ptolomeo del Sol respecto de la Latitud Celeste ( $x \in$ $\mathrm{M}(\mathrm{S} \beta S E D))$ syss

(1) $x \in \mathrm{M}(\mathrm{SED})$

(2) para todo $t \in T$

Si Sol $\in P l \& p u n(S o l)=s$, entonces: $\beta(s, t)=0$

\footnotetext{
${ }^{17}$ Puede ser tomada prestada de la matemática que la definiría con facilidad como puntos pertenecientes a la misma recta.

${ }^{18}$ Ver definición auxiliar 31 del apéndice 1.
} 


\subsubsection{Ley especial para el Sol respecto de la Longitud Celeste}

También puede establecerse una ley especial para el Sol, respecto de su longitud. La longitud celeste del Sol es 0 en uno de los puntos en los que la eclíptica se cruza con el ecuador celeste. Esto se produce en los equinoccios y longitud 0 corresponde al equinoccio de primavera (del hemisferio norte). El día de equinoccio de primavera (el de marzo), así como el de otoño (el de septiembre), el día y la noche duran exactamente lo mismo. Con ello podremos definir el instante de tiempo en el que se produce un equinoccio y en el que, por lo tanto, la longitud solar es 0 ó 180. Luego tendremos que distinguir entre los equinoccios. En el hemisferio norte, si en el día anterior al equinoccio, el día fue más largo que la noche, entonces estamos en el equinoccio de septiembre. Por ahora omitiremos el detalle que ello se cumple sólo en el hemisferio norte.

Lo primero que debemos definir, por lo tanto, es el instante en que amanece y aquel en que anochece. Al primero lo llamaremos "orto solar" (ORS) y al segundo "ocaso solar" (OCS). El orto solar es el subconjunto de $T$ en los que el centro del disco solar aparece en el horizonte y el ocaso el subconjunto que reúne los instantes en los que el centro del disco solar desaparece en el horizonte.

\section{Definición auxiliar 28 (orto solar):}

(1) $\mathrm{ORS}=\{t / t \in T \&$ si $\operatorname{pun}(\mathrm{Sol})=s$ en $t s$ aparece en el horizonte $\}$

\section{Definición auxiliar 29 (ocaso solar):}

(1) OCS $=\{t / t \in T \&$ si pun $(\mathrm{Sol})=s$ en $t s$ desaparece en el horizonte $\}$

Ahora la ley especial que dirá lo siguiente: tenemos cinco instantes de tiempo indexados del uno al cinco y dos con $x$ y $z$. Los cinco instantes serán el primer amanecer un día antes del equinoccio $\left(t_{1}\right)$, el anochecer de ese día $\left(t_{2}\right)$, al amanecer del día del equinoccio $\left(t_{3}\right)$, el anochecer de ese día $\left(t_{4}\right)$ y el amanecer del día siguiente al equinoccio $\left(t_{5}\right)$. Lo primero que diremos es que $t_{1}, t_{3}, t_{5}$ son tres ortos y que no hay ningún orto entre ellos, o sea, que son tres ortos seguidos. Ya sabemos que $t_{2}$ y $t_{4}$ están entre ellos, si además agregamos que son dos ocasos, es evidente que serán los ocasos intercalados entre esos amaneceres. Luego decimos que el día entre el amanecer $t_{3}$ y el anochecer $t_{4}$ duró lo mismo que la noche siguiente (entre $t_{4} \mathrm{y}_{5}$ ). Se trata, por lo tanto, de un equinoccio. El último requisito pide que el día anterior al equinoccio dure más que su noche correspondiente, por lo tanto, se trata de un equinoccio de primavera. Finalmente afirmamos que, si se dan esas condiciones, la longitud del mediodía del día del equinoccio será $0 .{ }^{19}$ Ver figura 5.

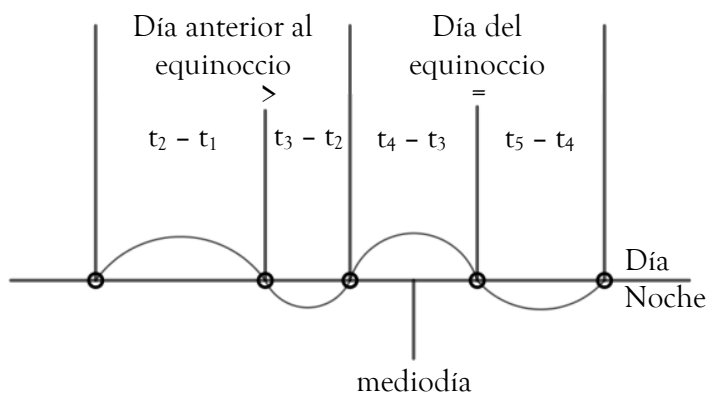

Figura 5.

\section{Definición 16:}

M(SגSED): $x$ es un sistema de epiciclos y deferente de Ptolomeo del Sol respecto de la Longitud Celeste ( $x \in$ $\mathrm{M}(\mathrm{S} \lambda \mathrm{SED}))$ syss

\footnotetext{
${ }^{19}$ En realidad para determinar exactamente el momento del equinoccio habría que utilizar un gnomo y medir la sombra que produce el Sol. Pero introducir todo ello volvería la presentación demasiado complicada. Suponemos arbitrariamente que el equinoccio se produce al mediodia.
} 
(1) $x \in \mathrm{M}(\mathrm{SED})$

(2) para todo $t \in T$

Si Sol $\in P l \& \operatorname{pun}(\mathrm{Sol})=s \& t_{1}, t_{2}, t_{3}, t_{4}, t_{5}, t_{x}, t_{z} \in T \& t_{1}, t_{3}, t_{5} \in$ ORS \& $\nexists t_{x}, t_{z} \in$ ORS tal que $t_{1}<t_{x}<t_{3}<t_{z}<t_{5} \& t_{2}, t_{4}, \in$ OCS \& $t_{4}-t_{3}=t_{5}-t_{4} \& t_{2}-t_{1}>t_{3}-t_{2}$, entonces: $\lambda\left(s,\left(t_{4}-t_{3}\right) / 2\right)=0$

\subsection{La Red Teórica del Sistema de Epiciclos y Deferentes de Ptolomeo}

La red teórica de SED podría expresarse como sigue. Hemos decidido, para que sea más clara, introducir la distinción entre los planetas que retrogradan y los que no, y los interiores y los exteriores, el Sol y la Luna aunque, por supuesto, no se trata de leyes, pero permite agruparlas de una manera más clara.

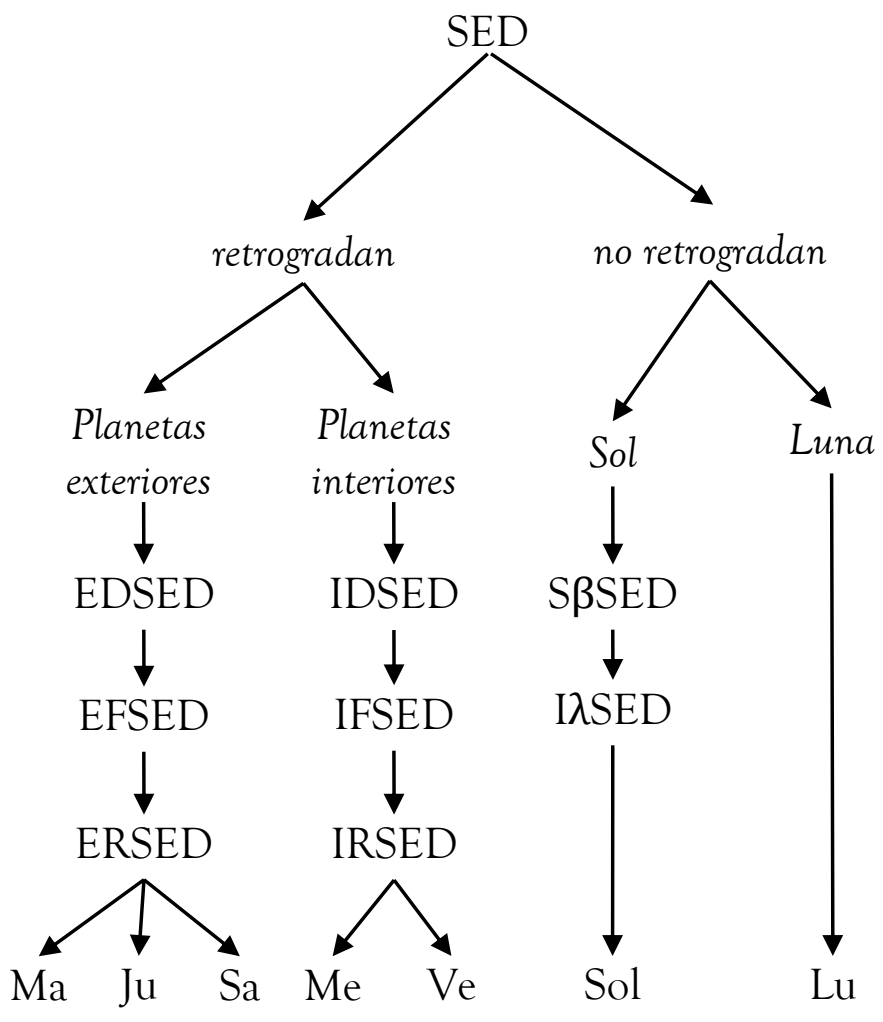

\section{Conclusión}

Hemos propuesto una reconstrucción del sistema de epiciclos y deferentes de Ptolomeo que, como decíamos en la introducción, es incompleta y perfectible, pero esperamos que sirva como primer paso.

\section{Apéndices}

4.1. Apéndice 1: Método de determinación de la longitud y latitud en función de las coordenadas polares

En la caracterización de la longitud celeste sólo hemos dicho que es una función continua entre [0-360] y que devuelve un grado, teniendo en el dominio un punto y un instante de tiempo. Utilizando las coordenadas polares es posible, sin embargo, introducir un método de determinación de la longitud. En efecto, la longitud puede ser interpretada como el segundo valor que devuelve una coordenada polar siempre que se cumplan tres condiciones: a) los puntos ( $p_{4}$ y $p_{5}$ ) que, junto con $p_{2}$, determinaban 
el plano de referencia pertenezcan al deferente del Sol, de tal manera que, entonces, ese plano sea el plano de la eclíptica; b) el polo desde el que se miden las coordenadas ( $\left.p_{2}\right)$ sea el centro de la Tierra y c) el punto que sirve para marcar el cero de la coordenada $\left(p_{3}\right)$ sea un punto en el que esté el Sol en un equinoccio de primavera (mejor: el punto del último equinoccio de primavera). En las mismas condiciones, la latitud corresponde al tercer valor de la imagen de las coordenadas polares.

Definición auxiliar 30 (método de determinación de la longitud celeste en función de co):

(1) para todo $t \in T$, si $t_{1}, t_{2}, t_{3} \in T \& p_{1}, p_{4}, p_{5} \in P \& g, h \in \mathbb{R}_{(0-360)} \& s \in \mathbb{R}^{+} \& \lambda\left(\right.$ pm(som(sol)), $\left.t_{1}\right)=0$

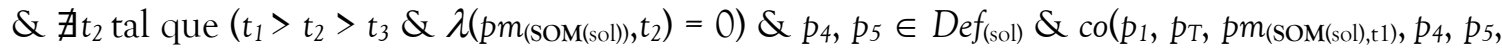
$\left.t_{3}\right)=(s, g, h)$, entonces $\lambda\left(p_{1}, t_{3}\right)=g$

Definición auxiliar 31 (método de determinación de la latitud celeste en función de co):

(1) para todo $t \in T$, si $t_{1}, t_{2}, t_{3} \in T \& p_{1}, p_{4}, p_{5} \in P \& g, h \in \mathbb{R}_{(0.360]} \& s \in \mathbb{R}^{+} \& \lambda\left(\right.$ pm(som(sol)), $\left.t_{1}\right)=0$

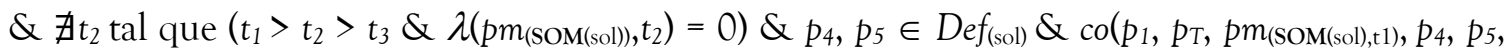
$\left.t_{3}\right)=(s, g, h)$, entonces $\beta\left(p_{1}, t_{3}\right)=h$

\subsection{Apéndice 2: Definición de la unidad de $\mathrm{S}$}

Como hemos dicho, por definición, el deferente de un determinado SOM mide 60 partes. Entonces:

Definición auxiliar 32 (partes en función del radio del deferente):

(1) Para todo $\left.t \in T, x \in P l, p_{1} \in \operatorname{Def}_{(x)} \rightarrow S\left(p_{1}, \mathcal{C}_{(\operatorname{Def}(x)}\right), x, t\right)={ }_{\text {def }} 60^{\mathrm{ps}}{ }_{(x)}$

\subsection{Apéndice 3: Demostración de la equivalencia de los grosores}

La equivalencia de los grosores puede demostrarse de la siguiente manera: sabemos por la definición de $\mathrm{GA}_{\text {som(pl) }}($ def. $\operatorname{aux} .21)$ que es igual a $\hat{S}_{m a ́ x}(\mathrm{pll}): \hat{S}_{\min (\mathrm{pl)})}$ También sabemos, por la segunda metrización de $\sigma\left(\right.$ def. aux. 18) que $\mathrm{S}\left(p_{1}, p_{T}, p l, t_{1}\right) \cdot K_{p l}=\hat{S}\left(p_{1}, p_{T}, t_{1}\right) y$, por lo tanto, que $\mathrm{GA}_{\mathrm{som}(\mathrm{pl})}=\operatorname{Smáx}(\mathrm{pl}) \cdot K_{p l}: \operatorname{Smin}(\mathrm{pl}) \cdot$ $K_{p l}$, cancelando $K_{p l}$ obtenemos que $G A_{\text {som(pl) }}=\operatorname{Smáx}(\mathrm{pl}): \operatorname{Smin}(\mathrm{pl})$, pero ello es justamente $G R_{\mathrm{som}(\mathrm{pl}) \text {, por lo }}$ que $\mathrm{GA}_{\mathrm{som}(\mathrm{pl})}=\mathrm{G} R_{\mathrm{som}(\mathrm{p}) \text {. }}$.

4.4. Apéndice 4: cómo obtener el valor de las distancias máximas y mínimas de todos los planetas a partir de la condición de ligadura y el DAL

Obtendremos, como ejemplo, el valor del perigeo del Sol de la siguiente manera:

$$
\hat{S}_{\min (\mathrm{Sol})}=\hat{S}_{\operatorname{máx}(\mathrm{Lu})} \cdot \mathrm{G} R_{\mathrm{som}(\mathrm{me})} \cdot \mathrm{GR} R_{\mathrm{som}(\mathrm{ve})}
$$

Ya que sabemos que:

$\hat{S}_{\min (\text { (Sol) }}=\hat{S}_{\max }(\mathrm{ve})$ ya que $\operatorname{ord}_{(\mathrm{sol})}=\operatorname{ord}_{(\mathrm{ve})}+1($ por C(SED) $)$

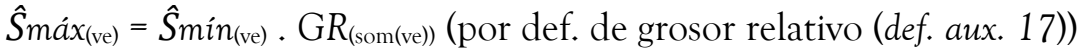

$\hat{S}_{\min }(\mathrm{ve})=\hat{S}_{\max }$ (me) ya que $\operatorname{ord}_{(\mathrm{ve})}=\operatorname{ord}_{(\mathrm{me})}+1($ por C(SED) $)$

$\hat{S}_{\max (\mathrm{me})}=\hat{S} \min (\mathrm{me}) \cdot G R_{(\mathrm{som}(\mathrm{me}))}$ (por def. de grosor relativo (def. aux. 17))

$\hat{S}_{\min (\mathrm{me})}=\hat{S}_{\max } x_{(\mathrm{lu})}$ ya que $\operatorname{ord}_{(\mathrm{lu})}=\operatorname{ord}_{(\mathrm{me})}+1(\operatorname{por} \mathrm{C}(\mathrm{SED}))$

Y $\hat{S}_{\text {máx(lu) }}$ lo hemos obtenido por $\mathrm{L}_{1}(\mathrm{SED})$

Por lo tanto: $\hat{S}_{\min (\text { (sol) }}=\hat{S}_{\operatorname{má}} x_{(\mathrm{ve})}=\hat{S}_{\min (\mathrm{ve})} \cdot G R_{(\mathrm{som}(\mathrm{ve}))}$

pero $\hat{S}_{\min }(\mathrm{ve})=\hat{S}_{\max }(\mathrm{me})$, por lo que:

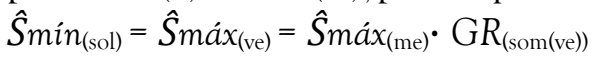

pero $\hat{S}_{\text {máx(me) }}=\hat{S} \hat{m i n}_{\text {(me) }} . G R_{(\text {som(me)), }}$ por lo que:

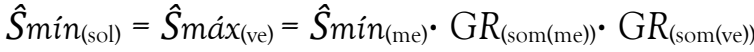

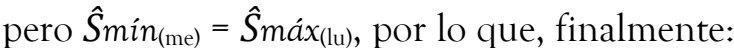

$\hat{S}_{\min (\mathrm{Sol})}=\hat{S}_{\operatorname{má} x_{(\mathrm{Lu})}} \cdot G R_{\mathrm{som}(\mathrm{me})} \cdot G R_{\mathrm{som}(\mathrm{ve})}, \mathrm{Q} \cdot$ E.D. 


\subsection{Apéndice 5: Otras definiciones}

Con el instrumental que hemos desarrollado se pueden definir varios conceptos importantes que pertenecen a la astronomía ptolemaica, algunos de los cuales detallamos a continuación. Todas están representadas en la figura 7 .

\subsubsection{Elongación}

La elongación de un planeta es la diferencia entre su longitud y la longitud del Sol. Ya la hemos utilizado al hablar de las retrogradaciones, pero ahora podemos definirla.

\section{Definición auxiliar 33 (elongación):}

(1) $\quad \eta^{\prime}: P l \times T \rightarrow \mathbb{R}_{(0-180)} \& \eta_{(p l, t)}=z$ syss para $t \in T$, si pun $(s o l)=s \& p u n(p l)=n,|\lambda(s, t)-\lambda(n, t)|=x \&$ si $x \leq 180$, entonces $z=x$, si $x>180$, entonces $z=x-180$

\subsubsection{Longitud media del Sol}

Ya la hemos utilizado también, es simplemente, la longitud del punto móvil de la órbita del Sol Medio. Ver figura 6.

Definición auxiliar 34 (longitud media del Sol):

(1) $\lambda m_{\text {(sol): }}$ Sol $\times T \rightarrow \mathbb{R}_{(0-360]} \& \lambda m_{(\mathrm{sol}, t)}={ }_{\text {def }} \lambda(p m(O(m), t))$

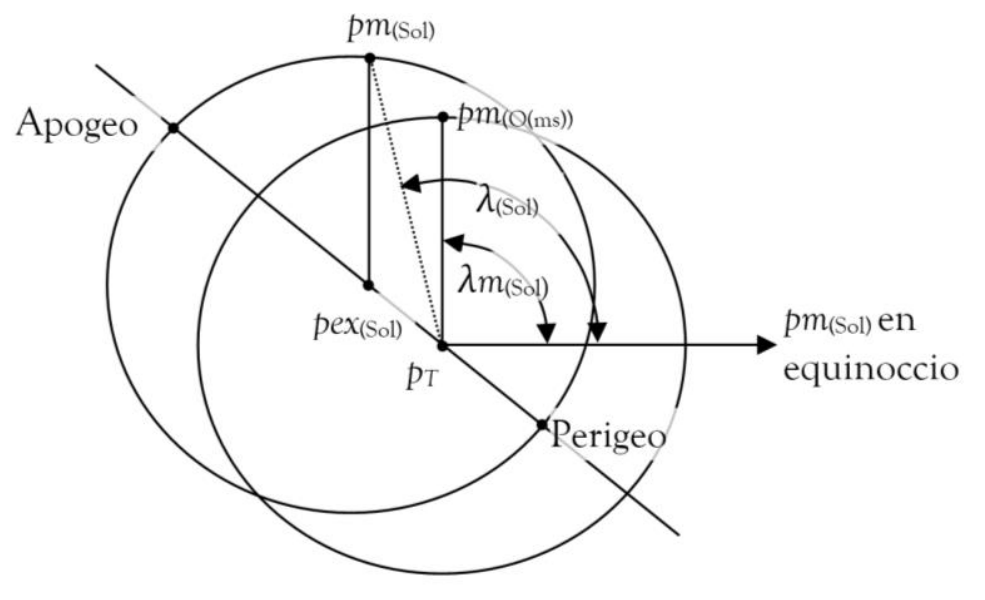

Figura 6.

4.5.3. Longitud media del resto de los planetas

En el resto de los planetas, la longitud media es la longitud que posee el punto móvil del deferente del planeta. Pero, en el caso de los planetas, no es estrictamente una "longitud" porque el polo de las coordenadas polares no es el centro de la Tierra $\left(p_{T}\right)$ sino el punto ecuante del deferente.

Definición auxiliar 35 ("longitud" media del resto de los planetas):

(1) $\lambda m_{\text {(pl-sol): }} P l_{\text {sol }} \times T \rightarrow \mathbb{R}_{(0-360]} \&$ si $t_{1}, t_{2}, t_{3} \in T \& g, h \in \mathbb{R}_{(0-360]} \& s \in \mathbb{R}^{+} \& p_{4}, p_{5} \in \operatorname{def}_{(\text {sol) }} \&$

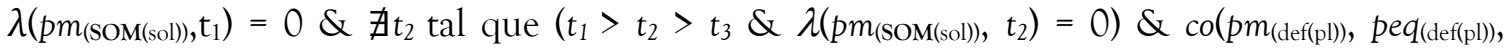
$\left.p m_{(\mathrm{SOM}(\mathrm{sol}), \mathrm{t} 1)}, p_{4}, p_{5}, t_{3}\right)=(s, g, h)$, entonces $\lambda m_{(\mathrm{pl}, \mathrm{t} 3)}=g$

\subsubsection{Longitud verdadera de un planeta}

La longitud verdadera es la longitud que debería ocupar el planeta, es decir, la del punto móvil del SOM de ese planeta.

Definición auxiliar 36 (longitud verdadera de un planeta): 
(1) $\quad \lambda v: P l \times T \rightarrow \mathbb{R}_{(0-360),} \& \lambda v_{(\mathrm{pl}, \mathrm{t})}={ }_{\mathrm{def}} \lambda(\operatorname{pm}(\mathrm{SOM}(\mathrm{pl}), \mathrm{t}))$

\subsubsection{Anomalía verdadera del epiciclo de un planeta}

La anomalía verdadera del epiciclo de un planeta expresa el movimiento del planeta sobre el epiciclo. Es, por lo tanto, el segundo valor de las coordenadas polares del punto móvil del epiciclo, cuando su polo es el centro del epiciclo. Lo difícil es determinar el punto con el que el polo forma el valor 0 para ese ángulo, ya que ese punto va cambiando. El epiciclo de un planeta tendrá argumento 0 cuando está alineado con el centro de la Tierra y el centro de su epiciclo y está "fuera" del deferente. Por lo tanto, pediremos que ese punto $\left(p_{a}\right)$ forme parte del epiciclo, esté alineado con el centro del epiciclo y el centro de la Tierra y, para que esté afuera, no forme parte de ninguna órbita con centro en el centro del deferente y de radio menor al radio de éste.

\section{Definición auxiliar 37 (anomalía verdadera del epiciclo de un planeta):}

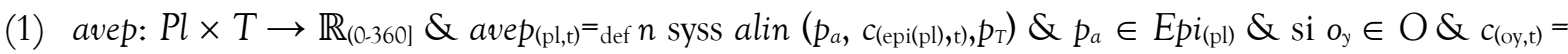
$c_{(\operatorname{def}(\mathrm{pl}), t)} \& r_{(\mathrm{oy})}<r_{(\operatorname{def}(\mathrm{pl})}$, entonces $p_{a} \notin o_{y} \& c_{0}\left(p_{(\text {epi(pl) }),} c_{(\text {epi }}(\mathrm{pll)}), p_{a}, p_{4}, p_{5}, t\right)=(r, n, h)$

\subsubsection{Anomalía media del epiciclo de un planeta}

La anomalía media de un epiciclo es exactamente igual a la verdadera, pero medida desde el punto ecuante del deferente y no desde el centro de la Tierra.

\section{Definición auxiliar 38 (anomalía media del epiciclo de un planeta):}

(1) amep: $P l \times T \rightarrow \mathbb{R}_{(0.360]} \&$ amep(pl,t) ${ }_{\text {def }} n$ syss $\exists o_{y}-r_{(\text {epi(pl) }} \& \operatorname{alin}\left(p_{a}, c_{(\text {epi }(p)), t)}, p e q_{(\operatorname{def}(p))}\right) \& p_{a} \in E p i_{(p l)}$

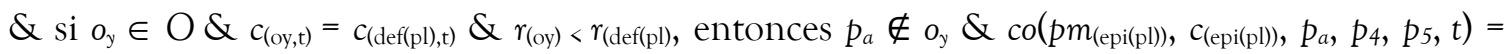
$(r, n, h)$

\subsubsection{Longitud del apogeo de un planeta}

La longitud del apogeo expresa la distancia angular entre el equinoccio de primavera y el punto en el que el planeta alcanza su apogeo (eso marca la línea apsidal). Ese punto está alineado con el centro de la Tierra y el centro del deferente. Hay dos puntos que, perteneciendo al deferente están alineados con el centro de la Tierra y el centro del deferente, pero el apogeo es el que se encuentra más alejado de la Tierra.

Definición auxiliar 39 (longitud del apogeo de un planeta):

(1) $\lambda a_{(\mathrm{pl}):} P l \times T \rightarrow \mathbb{R}_{(0-360)} \& \lambda a_{(\mathrm{pl}, \mathrm{t})}=_{\text {def }} \lambda_{(\mathrm{p} 1, \mathrm{t})} \operatorname{syss} p_{1}, p_{2} \in \operatorname{def}_{(\mathrm{pl})} \& \operatorname{alin}\left(p_{2}, p_{\mathrm{T}}, \operatorname{pex}_{\left.(\mathrm{def}(\mathrm{pl})), p_{1}\right) \& \text { para todo }}\right.$ $t \in T: S\left(p_{1}, p_{T}, p l, t\right)<S\left(p_{2}, p_{T}, p l, t\right)$ 


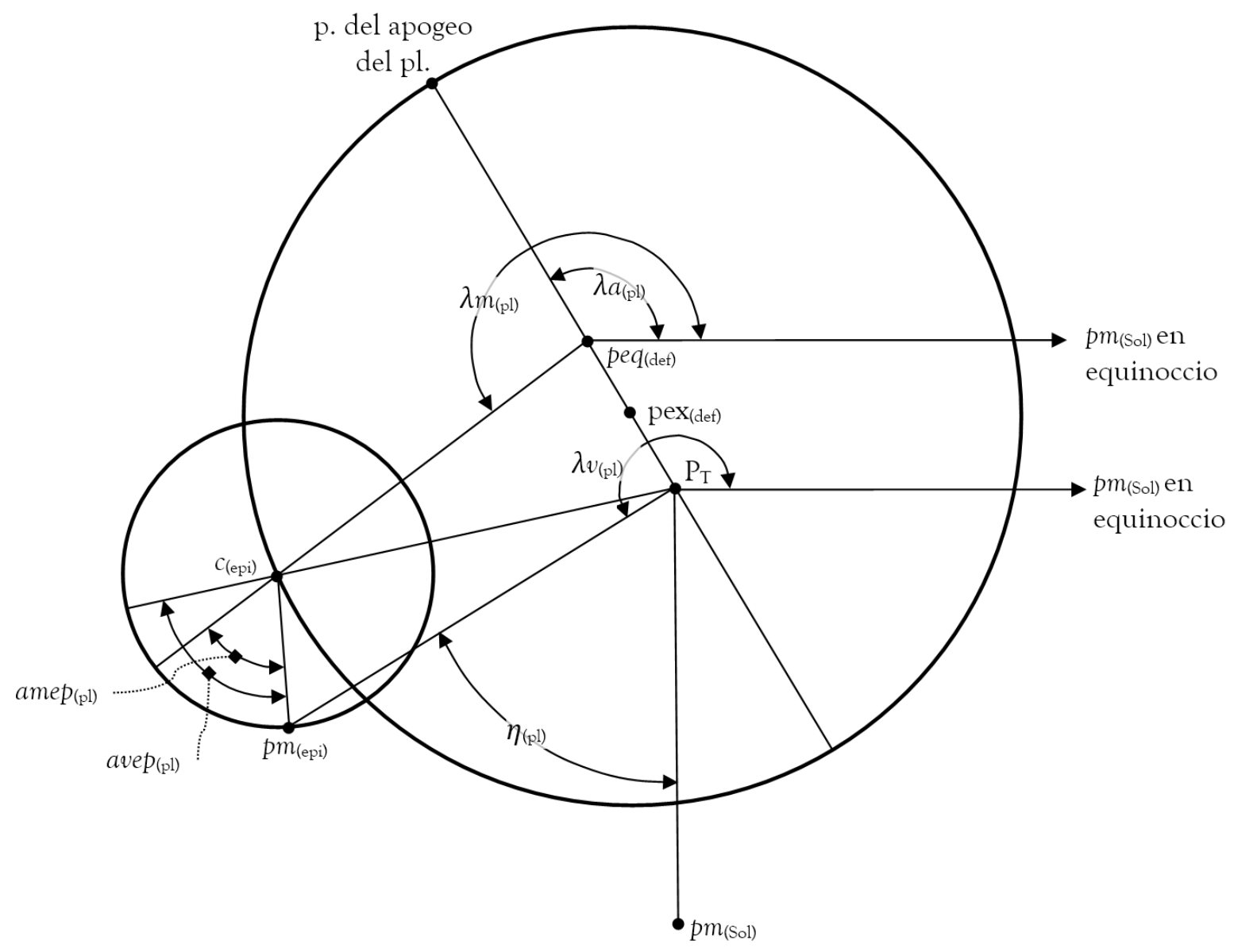

Figura 7.

Bibliografía

Balzer, W. (1997), Teorias empiricas: modelos, estructuras y ejemplos, Madrid: Alianza.

Evans, J. (1998), The History and Practice of Ancient Astronomy, Oxford: Oxford University Press.

Goldstein, B. R. (1967), "The Arabic Version of Ptolemy's Planetary Hypotheses", Transactions of the American Philosophical Society, New Series, Vol. 57, part. 4.

Heiberg, J. L. (ed.) (1898-1903), Claudii Ptolemaei Opera quae exstant omnia, vol. I, Syntaxis Mathematica, 2 vols., Leipzig: Teubner.

Heiberg, J. L. (ed.) (1907), Claudii Ptolemaei Opera quae exstant omnia, vol. II, Opera Astronomica Minora, Leipzig: Teubner.

Heidelberg, M. (1976), "Some Intertheoretic Relations between Ptolemean and Copernican Astronomy", Erkenntnis 10: 323-336

Neugebauer, O. (1975), A History of Ancient Mathematical Astronomy, 3 vols., Berlin: Springer.

Pedersen, O. (1974), "A Survey of the Almagest", Acta Historica Scientirarum Naturalium et medicinalium, vol. 30, Odense: Odense University Press.

Pérez Sedeño, E. (1987), Las Hipótesis de los Planetas (introducción y notas de Eulalia Pérez Sedeño; versiones castellanas de José García Blanco y Aurora Cano Ledesma), Madrid: Alianza.

Ptolemy, C., Almagest, en Tommer (1998) y Heiberg (1898-1903).

Toomer, G. J. (1998), Ptolemy's Almagest, Princeton, NJ: Princeton University Press, $1^{\mathrm{a}}$ ed. London: Durkworth, 1984. 